\title{
Uniqueness and the Global Markov Property for Euclidean Fields: The Case of General Exponential Interaction
}

\section{Bogusław Zegarliński}

Zentrum für Interdisziplinare Forschung, Universität Bielefeld, Wellinberg 1, D-4800 Bielefeld 1, Federal Republic of Germany

Abstract. The uniqueness and the global Markov property for the regular Gibbs measure corresponding to the interaction

$$
U_{A}(\varphi):=\lambda \int_{A} d_{2} x \int d \varrho(\alpha): e^{\alpha \varphi}:_{0}(x)
$$

[for $\lambda>0, d \varrho(\alpha)$ a probability measure with support in $(-2 \sqrt{\pi}, 2 \sqrt{\pi})]$ is proved.

\section{Introduction}

\subsection{Definitions and Notations}

By $\mathscr{F}$ we denote a family of bounded open sets in $\mathbb{R}^{d}$ partially ordered by the filtering inclusion relation $\leqq$ (i.e. $\Lambda_{1}, \Lambda_{2} \in \mathscr{F} \Rightarrow \exists \Lambda_{3} \in \mathscr{F}, \Lambda_{1} \leqq \Lambda_{3}, \Lambda_{2} \leqq \Lambda_{3}$ ).

By $\mathscr{F}_{0}:=\left\{\Lambda_{n} \in \mathscr{F}: \Lambda_{n} \subseteq \Lambda_{n+1}\right\}_{n \in \mathbb{N}}$ we denote a countable base of $\mathscr{F}$ (i.e. $\left.\forall \Lambda \in \mathscr{F}, \exists \Lambda_{n} \in \mathscr{F}_{0}, \Lambda \subseteq \Lambda_{n}\right)$. We always assume that boundary $\partial \Lambda$ of $\Lambda \in \mathscr{F}$ is piecewise-C $C^{1}$-curve. We assume that $\bigcup_{\mathscr{F}_{0}} \Lambda_{n}=\mathbb{R}^{d}$. We write $\Lambda^{c}:=\mathbb{R}^{d} \backslash \Lambda$ and
int $\Lambda:=\Lambda \supset \Lambda$.

Let $\mathscr{D} \equiv \mathscr{D}_{\text {real }}\left(\mathbb{R}^{d}\right)$ be the space of $C_{0}^{\infty}\left(\mathbb{R}^{d}\right)$ real functions and $\mathscr{S} \equiv \mathscr{S}_{\text {real }}\left(\mathbb{R}^{d}\right)$ the space of $C^{\infty}\left(\mathbb{R}^{d}\right)$ real rapidly decreasing functions, which are topologized as usually, e.g. [35, p. 28] for $\mathscr{D}$ and [35, p. 146] for $\mathscr{S}$. By $\mathscr{D}^{\prime}\left(\right.$ respectively $\left.\mathscr{S}^{\prime}\right)$ we denote the real topological dual space of $\mathscr{D}$ (respectively $\mathscr{S}$ ). For $\Lambda \subseteq \mathbb{R}^{d}$, we write $f \in \mathscr{D}_{\Lambda}$ (respectively $\left.\mathscr{S}_{\Lambda}\right)$ if $f \in \mathscr{D}$ (respectively $\mathscr{S}$ ) and $\sup f \subseteq \Lambda$. Let for $f \in \mathscr{D}$ (respectively $\mathscr{S}$ )

$$
\varphi(f): \mathscr{D}^{\prime} \rightarrow \mathbb{R}: \mathscr{D}^{\prime} \ni \eta \mapsto \varphi(f)(\eta):=\eta(f)
$$

(respectively for $\mathscr{S}^{\prime}$ we denote this function by the same letters). For arbitrary $\Lambda \cong \mathbb{R}^{d}$ open $\Sigma_{\Lambda}$ (respectively $\mathscr{B}_{\Lambda}$ ) is the smallest $\sigma$-algebra of subsets in $\mathscr{D}^{\prime}$ (respectively $\mathscr{S}^{\prime}$ ), such that all functions $\left\{\varphi(f): f \in \mathscr{D}_{A}\right\}$ (respectively $f \in \mathscr{S}_{A}$ ) are measurable. If $\Lambda=\mathbb{R}^{d}$ we write $\Sigma \equiv \Sigma_{\mathbb{R}^{d}}$ and $\mathscr{B} \equiv \mathscr{B}_{\mathbb{R}^{d}}$. For arbitrary $\Lambda \subseteq \mathbb{R}^{d}$ we define

$$
\Sigma_{\Lambda}:=\bigcap_{A \subset A^{\prime} \text { (open) }} \Sigma_{\Lambda} \quad\left(\text { respectively } \mathscr{B}_{\Lambda}:=\bigcap_{\Lambda \subset \Lambda^{\prime} \text { (open) }} \mathscr{B}_{\Lambda^{\prime}}\right) .
$$


If the function $F: \mathscr{D}^{\prime} \rightarrow \mathbb{R}$ (respectively $\mathscr{S}^{\prime} \rightarrow \mathbb{R}$ ) is $\Sigma, \Sigma_{\Lambda}$ (respectively $\mathscr{B}, \mathscr{B}_{\Lambda}$ ) measurable, we write $F \in \Sigma, F \in \Sigma_{\Lambda}$ (respectively for $\mathscr{B}, \mathscr{B}_{\Lambda}$ ).

Let $\mu, \mu_{0}$ be the probability measures on $\left(\mathscr{D}^{\prime}, \Sigma\right)$ [respectively $\left.\left(\mathscr{S}^{\prime}, \mathscr{B}\right)\right]$. By $\mu(F), \mu_{0}(F)$ we denote the expectations values of $F$ with the measures $\mu$ and $\mu_{0}$. For $F \in L_{1}(\mu)$ or $F \in L_{1}\left(\mu_{0}\right)$ by $E_{\mu}\left(F \mid \Sigma_{\Lambda}\right), E_{\mu_{0}}\left(F \mid E_{\Lambda}\right) \equiv E_{0, \Lambda}(F)$ we denote the conditional expectations of $F$ associated to $\mu$, respectively $\mu_{0}$ with respect to $\Sigma_{\Lambda}$ (analogously for $\mathscr{B}_{\Lambda}$ ). We write

$$
\mu\left(F, F^{\prime}\right):=\mu\left(F \cdot F^{\prime}\right)-\mu(F) \cdot \mu\left(F^{\prime}\right) .
$$

Let $\Delta$ (respectively $\Delta^{\partial \Lambda}$ ) be the selfadjoint Laplacean in $L_{2}\left(\mathbb{R}^{d}\right)$ with the essential domain $C_{0}^{\infty}\left(\mathbb{R}^{d}\right)$ [respectively Friedrichs extention in $L_{2}(\Lambda, d x)$ of $\Delta$ defined on $\left.C_{0}^{\infty}(\Lambda)\right]$. For arbitrary but fixed through all the paper $m_{0}>0$, by $G(x, y)$ [respectively $\left.G^{\partial \Lambda}(x, y)\right]$ we denote the kernel of the operator $\left(-\Delta+m_{0}^{2}\right)^{-1}$ in $L_{2}\left(\mathbb{R}^{d}\right)$ [respectively of $\left(-\Delta^{\partial \Lambda}+m_{0}^{2}\right)^{-1}$ in $\left.L_{2}(\Lambda, d x)\right]$. As usual

$$
\begin{aligned}
\|f\|_{-1}^{2} & :=\int d x d y f(x) G(x, y) f(y), \\
\|f\|_{-1, \partial \Lambda}^{2} & :=\int d x d y f(x) G^{\partial \Lambda}(x, y) f(y) .
\end{aligned}
$$

Let for $x \neq y$,

$$
K^{\partial \Lambda}(x, y):=G(x, y)-G^{\partial \Lambda}(x, y)
$$

and

$$
K^{\partial \Lambda}(x, x):=\lim _{y \rightarrow x} K^{\partial \Lambda}(x, y)
$$

The limit in (1.6) exists and $K^{\partial \Lambda}(x, x)$ is the continuous function in int $\Lambda$ (e.g. [3], [21, Chap. 7]).

0.2. Gibbs Measures ([10, 11, 15, 26, 30])

Definition 0.2.1. A local specification is a family $\mathscr{E} \equiv\left\{E_{\Lambda^{c}}\right\}_{\Lambda \in \mathscr{F}}$ whose elements are the functions

$$
E_{\Lambda^{c}}: \mathscr{D}^{\prime} \times \Sigma \rightarrow[0,1] \quad,(\Lambda \in \mathscr{F})
$$

with the following properties:

1) $\exists \Omega \in \Sigma, \forall \eta \in \Omega, E_{\Lambda^{c}}^{\eta}(\cdot)$ is a probability measure on $\left(\mathscr{D}^{\prime}, \Sigma\right)$, which restriction to $\Sigma_{\Lambda^{c}}$ coincides with the point measure $\delta_{\eta}$.

2) For $F \in \Sigma$ the function $\mathscr{D}^{\prime} \ni \eta \mapsto E_{\Lambda^{c}}^{\eta}(F)$ is $\Sigma_{\Lambda^{c}}$ measurable, being identically zero for $\eta \notin \Omega$.

3) The compatibility condition holds:

$$
\Lambda_{1}, \Lambda_{2} \in \mathscr{F}, \quad \Lambda_{1} \subset \Lambda_{2} \Rightarrow E_{\Lambda_{2}^{c}} E_{\Lambda_{1}^{c}}(\cdot)=E_{\Lambda_{2}^{c}}(\cdot) .
$$

Definition 0.2.2. A Gibbs measure for a local specification $\mathscr{E}$ is a probability measure on $\left(\mathscr{D}^{\prime}, \Sigma\right)$ such that

$$
\forall \Lambda \in \mathscr{F}, \quad \mu E_{\Lambda^{c}}(\cdot)=\mu(\cdot) .
$$

By $\mathscr{G}(\mathscr{E})$ we denote the set of all Gibbs measures for a specification $\mathscr{E}$ and by $\partial \mathscr{G}(\mathscr{E})$ the set of extreme points of $\mathscr{G}(\mathscr{E})$ [i.e. those $\mu \in \mathscr{G}(\mathscr{E})$ which cannot be represented as a convex combination of other Gibbs measures]. 
Remark. Equation (2.1) means that for each $F \in L_{1}(\mu)$,

$$
E_{\mu}\left(F \mid \Sigma_{A^{c}}\right)(\eta)=E_{\Lambda^{c}}^{\eta}(F) \quad \mu \text {-a.e. }
$$

for all $\Lambda \in \mathscr{F}$ (see [30]).

It is known, e.g. [15] that $\mu \in \mathscr{G}(\mathscr{E})$ defined on a standard Borel space can be uniquely represented as the integral over $\partial \mathscr{G}(\mathscr{E})$.

Definition 0.2.3. Let $F \in \Sigma_{\Lambda}(\Lambda \in \mathscr{F})$ be nonnegative:

i) If

$$
E_{\Lambda^{c}}(F) \in \Sigma_{\partial \Lambda}, \quad \forall \Lambda \in \mathscr{F},
$$

we say that $\mathscr{E}$ is Markov.

ii) If for the probability measure $\mu$ on $\left(\mathscr{D}^{\prime}, \Sigma\right)$

$$
E_{\mu}\left(F \mid \Sigma_{\Lambda^{c}}\right) \in \Sigma_{\partial \Lambda} \quad, \forall \Lambda \in \mathscr{F}
$$

we say that $\mu$ has the local Markov property.

iii) If for arbitrary open (with piecewise $C^{1}$ boundary) set $Q \subset \mathbb{R}^{d}$ and for arbitrary (nonnegative) $F \in \Sigma_{Q}$

$$
E_{\mu}\left(F \mid \Sigma_{Q^{c}}\right) \in \Sigma_{\partial Q},
$$

we say that $\mu$ has the global Markov property (GMP) and we write $\mu \in$ GMP.

If $\mu \in \mathscr{G}(\mathscr{E})$ and $\mathscr{E}$ is Markov then $\mu$ has the local Markov property, but it can happen that $\mu \notin \mathrm{GMP}$ (see e.g. the next section).

Definition 0.2.4. Let $\mathbb{T}_{I O(d)} \equiv\left\{T_{(R, a)}:(R, a) \in I O(d)\right\}$ be the representation of inhomogeneous Euclidean group $I O(d)$ in $d$ dimensions, whose action is defined on $\Sigma$ by the action on the functions $\varphi(f)$, as follows:

$$
T_{(R, a)} \varphi(f):=\varphi\left(f_{(R, a)}\right),
$$

where $f_{(R, a)}(x):=f\left(R^{-1}(x-a)\right)$,

i) $\mathscr{E}$ is Euclidean covariant if and only if

$$
\forall F \in \Sigma, \forall(R, a) \in I O(d), T_{(R, a)}\left(E_{A^{c}}(F)\right)=E_{((R, a) \Lambda)^{c}}\left(T_{(R, a)} F\right),
$$

where $(R, a) \Lambda:=\left\{x \in \mathbb{R}^{d}: R^{-1}(x-a) \in \Lambda\right\}$.

ii) A probability measure $\mu$ on $\left(\mathscr{D}^{\prime}, \Sigma\right)$ is called Euclidean invariant if and only if

$$
\forall(R, a) \in I O(d), \quad\left(T_{(R, a)}^{*} \mu\right)(F) \equiv \mu T_{(R, a)}(F)=\mu(F) .
$$

\subsection{The Euclidean Quantum Fields: The Exposition of Problems and Results}

One of the possible definitions of the notion: "the (scalar) quantum field with specified interaction," can be given (see $[12,24]$ ) in the Euclidean region with the aid of the notion of a Gibbs measure:

We say that a probability measure $\mu$ on $\left(\mathscr{D}^{\prime}, \Sigma\right)$ defines the (scalar) quantum field with the interaction $\left\{U_{\Lambda} \in \Sigma_{\Lambda}\right\}_{\Lambda \in \mathscr{F}}$ (with respect to the "free" measure $\mu_{0}$ ) if and only if

$$
\text { 1) } \forall \Lambda \in \mathscr{F}, \quad \quad \mu_{\mid \Sigma_{\Lambda}} \ll \mu_{0 \mid \Sigma_{\Lambda}} \text {, }
$$

i.e. $\mu$ is locally absolutely continuous with respect to $\mu_{0}$ and

$$
\mu \in \mathscr{G}(\mathscr{E})
$$


where the local specification $\mathscr{E}=\left\{E_{\Lambda^{c}}\right\}_{\Lambda \in \mathscr{F}}$ is given by

$$
E_{\Lambda^{c}}^{\eta}(\cdot):=\frac{E_{0, \Lambda^{c}}\left(e^{-U_{\Lambda}} \cdot\right)(\eta)}{E_{0, \Lambda^{c}}\left(e^{-U_{\Lambda}}\right)(\eta)},
$$

and

2) The Osterwalder-Schrader axioms [29] are satisfied by the moments of $\mu$ :

$$
\left\{\mu\left(\prod_{i=1}^{n} \varphi\left(f_{i}\right)\right), f_{i} \in \mathscr{D}, i=1, \ldots, n ; n \in \mathbb{N}\right\} .
$$

When the dimension of Euclidean space is $d=2$, the specification $\mathscr{E}$ in (3.2) can be defined with the aid of the "free" measure $\mu_{0}$ corresponding to the free (scalar) quantum field, i.e. Gaussian probability measure with mean zero and covariance $G(\cdot, \cdot)$, and the large class of interaction (additive) functionals $\left\{U_{\Lambda} \in \Sigma_{\Lambda}, \Lambda \in \mathscr{F}\right\}$ (at least for countable $\mathscr{F}$ ).

It is known (e.g. [1, 3]) that, under the general assumptions on the local specification (3.2), a Gibbs measure for $\mathscr{E}$ exists and in general it can be nonunique. The existence of many Gibbs measures for $\mathscr{E}$ can imply that many different quantum fields with the same interaction can exist; and moreover, that the vacuum vector is not cyclic for time-zero fields. The last is connected with the lack of GMP (2.5) for the Gibbs measure defining the quantum field. The GMP property is required if we want to reconstruct the quantum fields in the Minkowski region through Nelson's reconstruction theorem [28]. If the Gibbs measure has all the properties contained in Nelson's axioms (see [28]) besides GMP, we can reconstruct the quantum field in Minkowski space through the O-S reconstruction [29], but the time-zero fields have no vacuum as the cyclic vector. (For other consequences of GMP see [15] and refs. given there.)

Example. Now we will give the simple illustration to the uniqueness and GMP problems: Let $\mu_{0}$ be the Gaussian measure on $\left(\mathscr{D}^{\prime}, \Sigma\right)$ with mean zero and the covariance given by $G(\cdot, \cdot)$. Let $\mathscr{E}_{0}=\left\{E_{0, \Lambda^{c}}\right\}_{\Lambda \in \mathscr{F}}$ be the local specification given by [the extension to $\left(\mathscr{D}^{\prime}, \Sigma\right)$ of] a nice version of conditional expectations $E_{0, A^{c}}$ associated to $\mu_{0}$ and with respect to $\Sigma_{A^{c}}$. It is known (e.g. [13]), that $\mathscr{E}_{0}$ is well defined at least for a countable family $\mathscr{F}$ and that $\mathscr{E}_{0}$ is Markov [Definition 0.2.3i)].

As one can see, all measures in $\partial \mathscr{G}\left(\mathscr{E}_{0}\right)$ are indexed by the solution of the equation

$$
\left(-\Delta+m_{0}^{2}\right) \phi_{0}(x)=0, \quad x \in \mathbb{R}^{d}
$$

i.e.

$$
\phi_{0}(x)=\int d v(z) e^{m_{0} \sum_{i=1}^{d} z_{i} x_{i}}
$$

where $d v(z)$ is a bounded measure on the unit sphere in $\mathbb{R}^{d}$. In fact, we have

$$
\forall \mu \in \partial \mathscr{G}\left(\mathscr{E}_{0}\right), \quad \exists(\text { exactly one }) \phi_{0}, \quad \mu F(\varphi)=\mu_{0} F\left(\varphi+\phi_{0}\right) \equiv \mu_{\phi_{0}}(F) .
$$

There exists exactly one solution $\phi_{0} \equiv 0$, which has Euclidean symmetry, and the corresponding measure $\mu_{0}$ is the unique measure in $\mathscr{G}\left(\mathscr{E}_{0}\right)$ with the same symmetry. This is the unique measure with support in $\mathscr{S}^{\prime}$. 
Remark. In general, one can expect that the extremal Gibbs measures corresponding to the interaction of the form

$$
U_{\Lambda}(\varphi):=\lambda \int_{\Lambda} d x: V(\varphi):(x) \quad(\Lambda \in \mathscr{F})
$$

are indexed by the classical solutions of the equation

$$
\left(-\Delta+m_{0}^{2}\right) \phi_{0}(x)+\lambda V^{\prime}\left(\phi_{0}\right)(x)=0, \quad x \in \mathbb{R}^{d},
$$

and the measure $\mu_{\phi_{0}} \in \partial \mathscr{G}(\mathscr{E})$ corresponding to $\phi_{0}$ has the following form:

$$
\mu_{\phi_{0}} F(\varphi)=\mu_{\mathscr{S}^{\prime}, \phi_{0}} F\left(\varphi+\phi_{0}\right),
$$

where the measure $\mu_{\mathscr{S}^{\prime}, \phi_{0}}$ has support in $\mathscr{S}^{\prime}$. In particular, we have

$$
\mu_{\phi_{0}}(\varphi(f))=\mu_{\mathscr{S}^{\prime}, \phi_{0}}(\varphi(f))+\phi_{0}(f) .
$$

However, this correspondence cannot be, in general, one-to-one. It follows from the example of the : $\lambda \varphi^{4}+\sigma \varphi^{2}-h \varphi:_{2}$ model. If $\lambda>0, \sigma<0$ and $h$ is sufficiently small, then there are two solutions of the adequate equation (3.7), which have Euclidean symmetry, but there exists exactly one Euclidean invariant Gibbs measure (which is surely an extremal Gibbs measure; the proof of extremality exists only for the lattice version of this model, see [6,35]). It follows (see [18]) from analyticity of pressure in $h, \operatorname{Re} h \neq 0$ [23].

This phenomenon (the phase transition) is not, in general, well understood (at least by the author), although there exist interesting results concerning the problem (see $[14,25]$ and refs. therein).

The whole set $\mathscr{G}(\mathscr{E})$ is at least of mathematical interest, and in practice we consider those measures only which are regular in the sense that they are supported by $\mathscr{S}^{\prime}$.

Let us now discuss the GMP problem for the measures in $\mathscr{G}\left(\mathscr{E}_{0}\right)$ : We have

$$
\mu_{\phi_{0}} \in \partial \mathscr{G}\left(\mathscr{E}_{0}\right) \Rightarrow \mu_{\phi_{0}} \in \mathrm{GMP} .
$$

However, if we take for example two solutions of Eq. (3.3),

$$
\begin{aligned}
& \phi_{0}^{1}(x):=e^{m_{0} x_{1}}, \\
& \phi_{0}^{2}(x):=e^{-m_{0} x_{1}},
\end{aligned}
$$

then for the Gibbs measure

$$
\mu:=\frac{1}{2}\left(\mu_{\phi_{0}^{1}}+\mu_{\phi_{0}^{2}}\right),
$$

one can see that the GMP fails. (This example resembles in spirit that given by Goldstein in [22, Sect. 2].)

In general, we can expect that for Markov specification $\mathscr{E}$,

$$
\partial \mathscr{G}(\mathscr{E}) \subset \mathrm{GMP} .
$$

Now this is proved only for the dimension $d=1$, see [9].

The GMP problem for $\mu \in \mathscr{G}(\mathscr{E})$ can also be considered as the uniqueness problem for conditioned specification, i.e. is there, for any $Q \subset \mathbb{R}^{d}$ (open with smooth boundary $\partial Q$ ) and any $\eta \in \operatorname{supp} \mu$ (for $\mu$ - almost all $\eta$ ) only one Gibbs

1 See note added in proof on p. 221 
measure for the local specification

$$
\mathscr{E}_{Q, \eta}:=\left\{E_{\Lambda^{c}}^{\tilde{\eta}}\right\}_{\Lambda \in \mathscr{F} \cap Q, \tilde{\eta}_{\mid Q^{c}}=\eta_{\mid Q^{c}}} .
$$

Remark. This problem is connected with the uniqueness (in the supp $\mu$ ) of the solution of the Dirichlet problem in $Q$ with boundary condition in the support of $\mu$ for the classical Euclidean field equation (3.7).

In the paper [3] by Albeverio and Høegh-Krohn the uniqueness and GMP problems have been solved for Euclidean fields in two dimensions with the trigonometric interaction with respect to $\mu_{0}$ with $m_{0}>0$ :

$$
U_{\Lambda}(\varphi):=\lambda \int_{\Lambda} d_{2} x: \cos \alpha(\varphi+\theta):_{0}(x),
$$

with i) $\lambda>0$ sufficiently small,

ii) $\alpha \in(-\sqrt{2 \pi}, \sqrt{2 \pi})$. [The double dots in (3.14) mean the normal ordering with respect to $\mu_{0}$.]

The authors showed that there exists a unique Gibbs measure for the interaction (3.14), regular in the sense that

$$
\begin{aligned}
\mu_{\mid \mathscr{B}_{A}} & \ll \mu_{0_{\mathscr{O}_{A}}}, \quad \forall \Lambda \in \mathscr{F}, \\
\mu \varphi(f)^{2} & \leqq c\|f\|_{-1}^{2}
\end{aligned}
$$

( $c$ a positive constant) and that this (extremal) Gibbs measure has GMP.

Remark. The proof in [3] relies on the application of the cluster expansion (see [21, Chap. 18] and ref. [2] in our [3]) to the conditional measures. This expansion is uniformly - in volume and boundary conditions - convergent under the assumptions i) and ii) in (3.14). The existence theorem can be proven (see ref. [2] in [3]) by the use of the same technique for $\alpha$ 's in the larger interval $(-2 \sqrt{\pi}, 2 \sqrt{\pi})$. But in the case of the cluster expansion for conditional measures, in order to have the stability estimate (independent of boundary conditions) for the conditioned partition function in the unit cube by the method of J. Fröhlich (see ref. [22] in [3]), the restriction to the smaller interval $(-\sqrt{2 \pi}, \sqrt{2 \pi})$ is needed.

In our work we consider the exponential interaction (with respect to $\mu_{0}$ with $\left.m_{0}>0\right)$ :

$$
U_{\Lambda}(\varphi):=\lambda \int_{\Lambda} d_{2} x \int d \varrho(\alpha): e^{\alpha \varphi}:_{0}(x)
$$

where i) $\lambda>0$ arbitrary, ii) $d \varrho(\alpha)$ is a probability measure with support in $(-2 \sqrt{\pi}, 2 \sqrt{\pi})$. We distinguish the space $\mathscr{M}_{r}$ of probability measures $\mu$ on $\left(\mathscr{S}^{\prime}, \mathscr{B}\right)$, which fulfill the following conditions:

$$
\begin{aligned}
\mu_{\mid \mathscr{B}_{A}} & \ll \mu_{0_{\mid \mathscr{B}_{A}}, \quad \forall \Lambda \in \mathscr{F},}, \\
\mu e^{\varphi(f)} & \leqq a e^{\frac{1}{2}\|f\|^{2}-1}+b\|G \times f\|_{L_{1}},
\end{aligned}
$$

$a>0, b \geqq 0$ arbitrary, $f$ for which the right-hand side exists. The elements of $\mathscr{M}_{r}$ we will call the regular measures. (They are also regular, see Lemma 1.1.1, in the sense of (3.15) used in [3].) The definition (3.17) appears to be a natural consequence of one of our technical results (exp bound in Sect. 1.4). It can be shown - using

1 See note added in proof on p. 221 
Dobrushin's compactness arguments (e.g. [10, 11 and 6, 7]) - that in the space $\mathscr{M}_{r}$ there exists a Gibbs measure $\mu$ for $\mathscr{E}$, where $\mathscr{E}$ is given by (3.2) and (3.16). In fact, the measure $\mu$ is unique in $\mathscr{G}(\mathscr{E}) \cap \mathscr{M}_{r}$. It can be obtained exactly as in $[2,17]$ as the thermodynamic limit of finite volume measures with half-Dirichlet boundary condition. Such a measure defines the Euclidean field theory [with the mass gap $\geqq m_{0}$ for any measure $d \varrho(\alpha)$ in (3.16ii), which follows from one of our technical results: exponential clustering in Sect. 1.5]. We have also that $\mu \in$ GMP. The proof that $\mu \in \mathrm{GMP}$ is given as the proof of uniqueness for conditioned specification (3.13).

The technique of our proofs is based on the correlation inequalities, so we do not need the restriction of the interval of $\alpha$ 's as in [3].

The uniqueness and GMP problems for the interactions of the form (3.16) but with suppd $\varrho \subset(0, \varepsilon), \varepsilon$ small and $\varepsilon<2 \sqrt{\pi}$ have been also considered in [19]. We do the needed improvements of some lemmas. In our proofs of uniqueness we use also a different idea - of the FKG order (see e.g. [30]). Using this idea we prepare the extension of our results - in particular, the proof of GMP based on the method used in $[22,16,35]$ - to the case of the interactions (3.16) with the additional term $\sigma: \varphi^{2}:$ with $\sigma<0$ and $|\sigma|$ big.

Remark. The problems of GMP (and also of uniqueness) have been also considered in the following papers:

a) for lattice finite spin systems: [5, 22, 27] (implicitly also in [32]),

b) for lattice infinite spin systems: [31, 7, 6, 35],

c) for classical gases: [33, 22, 20],

d) solved under the general assumptions in one dimension in [9] (see also refs. therein for preceding particular results).

In the next section we present our technical results. The main ones are: FKG order in the set of conditioned measures (Sect. 1.3), the exponential bound (Sect. 1.4) and uniform - in volume and boundary condition - exponential clustering (Sect. 1.5) for conditioned measures.

The proofs of uniqueness (here it implies extremality) of the regular Gibbs measure is given in Sect. 2 and of the GMP in Sect. 3.

Summing up, our main result is:

Proposition. Let $\mathscr{E}=\left\{E_{\Lambda^{c}}^{\eta}\right\}_{\Lambda \in \mathscr{F}}$ be the local specification given by

$$
E_{\Lambda^{c}}^{\eta}(\cdot):=\frac{E_{0, \Lambda^{c}}\left(e^{-U_{\Lambda} \cdot}\right)(\eta)}{E_{0, \Lambda^{c}}\left(e^{-U_{\Lambda}}\right)(\eta)},
$$

where the interaction (with respect to the free measure $\mu_{0}$ with mass $m_{0}>0$ ) has the form:

$$
U_{\Lambda}(\varphi):=\lambda \int_{\Lambda} d_{2} x \int d \varrho(\alpha): e^{\alpha \varphi}:_{0}(x)
$$

with $\lambda>0$ arbitrary and $d \varrho(\alpha)$ a probability measure with support in $(-2 \sqrt{\pi}, 2 \sqrt{\pi})$.

Let $\mathscr{M}_{r}$ be the space of regular measures defined, by (3.17). Then:

1) There exists exactly one point $\mu$ in $\mathscr{G}(\mathscr{E}) \cap \mathscr{M}_{r}$,

2) $\mu \in \mathrm{GMP}$. 


\section{The Technicalities}

\section{1 .}

Let $C$ be a piecewise $C^{1}$-curve in $\mathbb{R}^{2}$. Let for $g \in C_{0}\left(\mathbb{R}^{2}\right)$. By $\psi_{g}^{c}(x)$ denote the unique solution of Dirichlet's problem:

$$
\begin{aligned}
& \left(-\Delta+m_{0}^{2}\right) \psi_{g}^{c}(x)=0 \quad \text { for } \quad x \in \mathbb{R}^{2} \backslash C, \\
& \psi_{g}^{c}(z)=g(z) \text { for } z \in C .
\end{aligned}
$$

It is known (e.g. [8]), that $\psi_{g}^{c}(x)$ exists and for fixed $x \in \mathbb{R}^{2} \backslash C$ the function $C_{0}\left(\mathbb{R}^{2}\right) \ni g \mapsto \psi_{g}^{c}(x)$ is positive, continuous and linear. The measure on $C$ corresponding to this functional is absolutely continuous with respect to the natural measure $d z$ on $C$, i.e.

$$
\psi_{g}^{c}(x)=\int_{c} d z P_{c}(x, z) g(z),
$$

where the Poisson kernel $P_{c}(x, z)$ is the measurable function on $\mathbb{R}^{2} \times C$.

Lemma 1.1.1. If $\mu \in \mathscr{M}_{r}$ then there exists $c>0$ such that

$$
\mu \varphi(f)^{2} \leqq c\|f\|_{-1}^{2}, \quad f \in C_{0}^{\infty}\left(\mathbb{R}^{2}\right) .
$$

So for each $x \notin C$ there exists an extension of the functional (1.2) to the function

$$
\mathscr{S}^{\prime} \ni \eta \mapsto \psi_{\eta}^{c}(x)
$$

for which $e^{\psi_{\eta}^{c}(x)} \in L_{p}(\mu)$ for $1 \leqq p<\infty$.

Proof. From the definition of $\left.\mathscr{M}_{r}[3.17,2)\right]$ we have for arbitrary $t>0$,

$$
\mu \varphi(f)^{2} \leqq \frac{1}{t^{2}} \mu\left(e^{t \varphi(f)}+e^{-t \varphi(f)}\right) \leqq \frac{2 a}{t^{2}} e^{\frac{t^{2}}{2}\|f\|^{2-1}+b t\|G \times f\|_{L_{1}}}
$$

Taking $t=\left(\frac{1}{2}\|f\|_{-1}^{2}+b^{2}\|G \times f\|_{L_{1}}^{2}\right)^{-1 / 2}$, we have for some constant $c_{1}>0$,

$$
\mu \varphi(f)^{2} \leqq c_{1}\left(\frac{1}{2}\|f\|_{-1}^{2}+b^{2}\|G \times f\|_{L_{1}}^{2}\right) .
$$

But

$$
\|G \times f\|_{L_{1}} \leqq\|G \times f\|_{L_{2}} \leqq\left\|G^{1 / 2}\right\|\left\|G^{1 / 2} f\right\|_{L_{2}}=\frac{1}{m_{0}}\left\|G^{1 / 2} f\right\|_{L_{2}} \equiv \frac{1}{m_{0}}\|f\|_{-1},(1
$$

where $\left\|G^{1 / 2}\right\|$ is the norm of operator $G^{1 / 2}$ in $L_{2}\left(\mathbb{R}^{2}, d_{2} x\right)$. Hence from (1.6) and (1.7) the bound (1.3) follows with some constant $c>0$. Now let $\left\{\chi_{\Lambda}, \Lambda \in \mathscr{F}_{0}\right\}$ be a sequence of characteristic functions and let $h_{\varepsilon} \in C_{0}^{\infty}\left(\mathbb{R}^{2}\right), h_{\varepsilon} \geqq 0, \int h_{\varepsilon}(x) d_{2} x=1$ be a sequence which goes (in $\mathscr{S}^{\prime}$ ) to the $\delta$ distribution as $\varepsilon \rightarrow 0$. Let us define the functions

$$
\mathscr{S}^{\prime} \ni \eta \mapsto \int_{c} d z P_{c}(x, z) \chi_{\Lambda}(z) \eta_{\varepsilon}(z) \equiv \psi_{\eta_{\varepsilon \mid \Lambda}}^{c}(x) ; \quad x \notin C,
$$

where $\eta_{\varepsilon}(z):=\eta\left(h_{\varepsilon}(\cdot-z)\right)$.

Now using (1.3) we can prove, by the arguments of [3] that the sequence of functions (1.8) converges in $L_{2}(\mu)$, as $\Lambda \nearrow \mathbb{R}^{2}$ [to the element $\psi_{\eta_{\varepsilon}}^{c}(x)$ ] and then as $\varepsilon \rightarrow 0$. Because the sequence $e^{\psi \varphi_{\varepsilon \mid \Lambda}^{c}(x)}(x \notin C)$ has uniformly in $\varepsilon$ and $\Lambda$ bounded $L_{p}(\mu)$ 
norms, hence using the $L_{2}(\mu)$ convergence of $\psi_{\eta_{\varepsilon \mid A}}^{c}(x)$ we can prove $L_{p}(\mu)$ convergence of $e^{\psi_{\eta_{\varepsilon \mid \Lambda}}^{c}(x)}$ as $\Lambda \nearrow \mathbb{R}^{2}$ to $e^{\psi_{\eta_{\varepsilon}}^{c}(x)}$ and to $e^{\psi_{\tilde{\eta}}(x)}$ as $\varepsilon \rightarrow 0$.

Now using (1.3) we have for $\mu \in \mathscr{M}_{r}$ the following:

Lemma 1.1.2 (see [3, Theorem 2.1 and Theorem 2.2]). Let $\mathscr{F}_{0}=\left\{\Lambda_{n} \in \mathscr{F}\right\}_{n \in \mathbb{N}}$ be $a$ countable base of $\mathscr{F}$ and let $\left\{\Omega_{n} \in \mathscr{F}: \Omega_{n} \subset \Lambda_{n}\right\}_{n \in \mathbb{N}}$ be another countable base of $\mathscr{F}$ such that $d\left(, \partial \Omega_{n}\right)=\frac{1}{2} d\left(0, \partial \Lambda_{n}\right) \underset{n \rightarrow \infty}{\longrightarrow} \infty$ and $\left|\Omega_{n}\right| \leqq \mathcal{O}\left(d\left(0, \partial \Omega_{n}\right)^{k}\right),\left|\Lambda_{n}\right| \leqq \mathcal{O}\left(d\left(0, \partial \Lambda_{n}\right)^{k}\right)$, where $k$ is a positive constant. then:

Let $\mu$ be a probability measure on $\left(\mathscr{S}^{\prime}, \mathscr{B}\right)$ which satisfies (1.3). Let $0<m<m_{0}$,

i) There is a subsequence $\left\{\Omega_{n^{\prime}}, \Lambda_{n^{\prime}}\right\}_{n^{\prime} \in \mathbb{N}}$ such that

$$
\lim _{n^{\prime} \rightarrow \infty} \sup _{x \in \Omega_{n^{\prime}}} e^{\frac{m}{2} d\left(\Omega_{n^{\prime}}, \partial \Lambda_{n^{\prime}}\right)}\left|\psi_{\eta}^{\partial \Lambda_{n^{\prime}}}(x)\right|=0, \quad \mu \text {-a.e. }
$$

ii) For any open $Q \subset \mathbb{R}^{d}$ with piecewise $C^{1}$-boundary $\partial Q$ there is a subsequence $\left(\Omega_{n^{\prime}}, \Lambda_{n^{\prime}}\right)_{n^{\prime} \in \mathbb{N}}$ (we assume that $\partial Q \cap \partial \Lambda_{n^{\prime}}$ and $\partial Q \cap \partial \Omega_{n^{\prime}}$ are finite point sets) such that

$$
\lim _{n^{\prime} \rightarrow \infty} \sup _{x \in \Omega_{n^{\prime}} \cap Q} e^{\frac{m}{2} d\left(\Omega_{n^{\prime}}, \partial \Lambda_{n^{\prime}}\right)}\left|\psi_{\eta}^{\partial Q}(x)-\psi_{\eta}^{\partial\left(Q \cap \Lambda_{n^{\prime}}\right)}(x)\right|=0, \quad \mu \text {-a.e. }
$$

\subsection{The Correlation Inequalities for Conditioned Measures}

By $\psi_{\eta} \equiv \psi_{\eta}(\alpha, x)$ we'll denote a random variable on $\left(\mathscr{S}^{\prime}, \mathscr{B}\right)$ being a function in $\alpha \in(-2 \sqrt{\pi}, 2 \sqrt{\pi})$ and $x \in \operatorname{int} \Lambda$, which is one of the following: $\alpha \psi_{\eta}^{\partial \Lambda}(x), \pm \alpha\left|\psi_{\eta}^{\partial \Lambda}(x)\right|$, $-\left|\alpha \psi_{\eta}^{\partial \Lambda}(x)\right|, \quad \alpha \psi_{\eta}^{\partial Q}(x), \quad \alpha \psi_{\eta}^{\partial Q}(x) \pm \alpha\left|\psi_{\eta}^{\partial Q}(x)-\psi_{\eta}^{\partial \Lambda \cap Q}(x)\right|, \quad \alpha \psi_{\eta}^{\partial Q}(x)-\mid \alpha\left(\psi_{\eta}^{\partial Q}(x)\right.$ $\left.-\psi_{\eta}^{\partial \Lambda \cap Q}(x)\right) \mid$ or convex linear combinations of them or identically zero.

Let

$$
\begin{aligned}
U_{\Lambda}\left(\varphi, \psi_{\eta}\right): & =\lambda \int_{\Lambda} d_{2} x \int d \varrho(\alpha) e^{\psi_{\eta}(\alpha, x)}: e^{\alpha \varphi}:_{0}(x) \\
& =\lambda \int_{\Lambda} d_{2} x \int d \varrho(\alpha) e^{\psi_{\eta}(\alpha, x)-\frac{\alpha^{2}}{2} K_{(x, x)}^{\partial A}}: e^{\alpha \varphi}:_{\partial \Lambda}(x),
\end{aligned}
$$

where $\Lambda \in \mathscr{F}, d \varrho(\alpha)=d \varrho_{+}(\alpha)+d \varrho_{-}(\alpha)$ is a probability measure with supp $d \varrho_{ \pm}(\alpha)$ $C[0, \pm 2 \sqrt{\pi})$ and : $:_{\partial \Lambda}$ means the normal ordering with respect to $\mu_{0}^{\partial \Lambda}$.

We define the conditioned measures

$$
\mu_{\Lambda, \psi_{\eta}}(\cdot):=\frac{\mu_{\partial}^{\partial \Lambda}\left(e^{-U_{\Lambda}\left(\varphi, \psi_{\eta}\right)} \cdot\right)}{\mu_{0}^{\partial \Lambda}\left(e^{-U_{\Lambda}\left(\varphi, \psi_{\eta}\right)}\right)} .
$$

In order to prove the correlation inequalities for these measures we define (following the idea of [19]) the measures $\mu_{\Lambda, \psi_{\eta_{\varepsilon}}}$ analogously as in (2.2) but with $\eta_{\varepsilon}(z):=\eta\left(h_{\varepsilon}(\cdot-z)\right)$ instead of $\eta$, and their lattice approximations (e.g. $\left.[17,21,24]\right)$

$$
\mu_{\Lambda, \psi_{\eta_{\varepsilon}}, \delta}(\cdot):=\frac{\mu_{0, \delta}^{\partial \Lambda}\left(e^{-U_{\Lambda, \delta}\left(\varphi_{\delta}, \psi_{\eta_{\varepsilon}}\right)} \cdot\right)}{\mu_{0, \delta}^{\partial \Lambda}\left(e^{-U_{\Lambda, \delta}\left(\varphi_{\delta}, \psi_{\eta_{\varepsilon}}\right)}\right)}
$$

where

$$
U_{\Lambda, \delta}\left(\varphi_{\delta}, \psi_{\eta_{\eta}}\right):=\lambda \delta^{2} \sum_{n \delta \in \Lambda} \int d \varrho(\alpha) e^{\psi_{\eta}(\alpha, x)-\frac{\alpha^{2}}{2} K^{\partial \Lambda}(n \delta, n \delta)}: e^{\alpha \varphi_{\delta}(n)}: \partial \Lambda
$$


with $n \in \mathbb{Z}^{2}, \varphi_{\delta}(n)=\varphi\left(f_{n, \delta}\right)$ for an appropriate element $f_{n, \delta} \in \mathscr{H}_{-1}$ and measure $\mu_{0, \delta}^{\partial \Lambda}$ is the restriction of $\mu_{0}^{\partial \Lambda}$ to the $\sigma$-algebra generated by $\left\{\varphi_{\delta}(n)\right\}_{n \delta \in \Lambda}$. We prove the convergence of $\mu_{\Lambda, \psi_{\eta_{\varepsilon}}, \delta}$ to $\mu_{\Lambda, \psi_{\eta_{\varepsilon}}}$ as $\delta \rightarrow 0$ and then the convergence of $\mu_{\Lambda, \psi_{\eta_{\varepsilon}}}$ to $\mu_{\Lambda, \psi_{\eta}}$ as $\varepsilon \rightarrow 0$. By this procedure the correlation inequalities, which holds for lattice measures, are carried out on the measures $\mu_{\Lambda, \psi_{\eta}}$.

Lemma 1.2.1. Let $\mu \in \mathscr{M}_{r}$ and $\Lambda \in \mathscr{F}$. Then

1. $\forall \eta \in \mathscr{S}^{\prime}, \forall \varepsilon>0$,

i) $U_{\Lambda}\left(\varphi, \psi_{\eta_{\varepsilon}}\right), U_{\Lambda, \delta}\left(\varphi_{\delta}, \psi_{\eta_{\varepsilon}}\right) \in L_{2}\left(\mu_{0}^{\partial \Lambda}\right), \mu_{\Lambda, \psi_{\eta_{\varepsilon}}}, \mu_{\Lambda, \psi_{\eta_{\varepsilon}}, \delta}$ are well defined.

ii) $U_{\Lambda, \delta}\left(\varphi_{\delta}, \psi_{\eta_{\varepsilon}}\right) \underset{\delta \rightarrow 0}{\longrightarrow} U_{\Lambda}\left(\varphi, \psi_{\eta_{\varepsilon}}\right)$ in $L_{2}\left(\mu_{0}^{\partial \Lambda}\right)$.

iii) For all $F \in L_{p}\left(\mu_{0}^{\partial \Lambda}\right), \quad 1 \leqq p \leqq \infty, \quad \mu_{\Lambda, \psi_{\eta_{\varepsilon}}, \delta} F\left(\varphi_{\delta}\right) \underset{\delta \rightarrow 0}{\longrightarrow} \mu_{\Lambda, \psi_{\eta_{\varepsilon}}} F(\varphi) \quad$ if $F\left(\varphi_{\delta}\right) \underset{\delta \rightarrow 0}{\longrightarrow} F(\varphi)$ in $L_{1}\left(\mu_{0}^{\partial \Lambda}\right)$ $\eta \in \mathscr{S}^{\prime}$.

2. i) $U_{\Lambda}\left(\varphi, \psi_{\eta}\right) \in L_{2}\left(\mu_{0}^{\partial \Lambda} \otimes \mu\right)$ and the measure $\mu_{\Lambda, \psi_{\eta}}$ are well defined for $\mu$-a.a.

ii) $U_{\Lambda}\left(\varphi, \psi_{\eta_{\varepsilon}}\right) \underset{\varepsilon \rightarrow 0}{\longrightarrow} U_{\Lambda}\left(\varphi, \psi_{\eta}\right)$ in $L_{2}\left(\mu_{0}^{\partial \Lambda} \otimes \mu\right)$.

iii) $\mu_{\Lambda, \psi_{\eta_{\varepsilon}}}(F) \underset{\varepsilon \rightarrow 0}{\stackrel{\varepsilon \rightarrow 0}{\longrightarrow}} \mu_{\Lambda, \psi_{\eta}}(F)$ for all $F \in L_{p}\left(\mu_{0}^{\partial \Lambda}\right), 1 \leqq p \leqq \infty$ and for $\mu$-a.e. $\eta \in \mathscr{S}^{\prime}$.

Proof. 1. Because for $\varepsilon>0$ the function $e^{\psi_{\eta_{\varepsilon}}(\alpha, x)-\frac{\alpha^{2}}{2} K^{\partial \Lambda}(x, x)}$ is continuous bounded the first part follows from [2] and [17] (at least for rectangles).

2. i) The fact that $U_{\Lambda}\left(\varphi, \psi_{\eta}\right)$ have the bounded $L_{2}\left(\mu_{0}^{\partial \Lambda} \otimes \mu\right)$ norm follows from the definition of regularity of $\mu$ by simple computations in which we use the expressions for $\mathscr{H}_{-1}$ - scalar products of Poisson kernels given in [3] (before Lemma 2.2). The restriction supp $d \varrho(\alpha) \subset(-2 \sqrt{\pi}, 2 \sqrt{\pi})$ is essential.

In order to prove that $\mu_{\Lambda, \psi_{\eta}}$ is well defined it suffices to prove that the denominator in (2.2) is different from zero for $\mu$-a.a. $\eta \in \mathscr{S}^{\prime}$. From Jensen's inequality we have

$$
\mu_{0}^{\partial \Lambda} e^{-U_{\Lambda}\left(\varphi, \psi_{\eta}\right)} \geqq e^{-\mu_{0}^{\partial \Lambda} U_{\Lambda}\left(\varphi, \psi_{\eta}\right)},
$$

and from regularity of $\mu$ and the definition of $\psi_{\eta}(\alpha, x)$

$$
\mu_{0}^{\partial \Lambda} U\left(\varphi, \psi_{\eta}\right)=\lambda \int_{\Lambda} d_{2} x d \varrho(\alpha) e^{\psi_{\eta}(\alpha, x)-\frac{\alpha^{2}}{2} K^{\partial \lambda}(x, x)} \in L_{1}(\mu),
$$

so is $\mu$-a.e. finite, hence (2.5) is greater than zero for $\mu$-a.a. $\eta \in \mathscr{S}^{\prime}$.

ii) Let

$$
U_{\Lambda}^{(n)}\left(\varphi, \psi_{\eta_{\varepsilon}}\right):=\lambda \int_{\Lambda} d_{2} x \int d \varrho(\alpha) \frac{\left(\psi_{\eta_{\varepsilon}}(\alpha, x)\right)^{n}}{n !}: e^{\alpha \varphi}:_{0}(x)
$$

then

$$
U_{\Lambda}\left(\varphi, \psi_{\eta_{\varepsilon}}\right)=\sum_{n=0}^{\infty} U_{\Lambda}^{(n)}\left(\varphi, \psi_{\eta_{\varepsilon}}\right)
$$

and for each $n \in \mathbb{N}$

$$
U_{\Lambda}^{(n)}\left(\varphi, \psi_{\eta_{\varepsilon}}\right) \underset{\varepsilon \rightarrow 0}{\longrightarrow} U_{\Lambda}^{(n)}\left(\varphi, \psi_{\eta}\right)
$$


in $L_{2}\left(\mu_{0}^{\partial \Lambda} \otimes \mu\right)$ which follows from the convergence of $\psi_{n_{\varepsilon}}(\alpha, x) \underset{\varepsilon \rightarrow 0}{\longrightarrow} \psi_{\eta}(\alpha, x)$ in $L_{p}(\mu), 1 \leqq p<\infty$ [and the explicit bound on $\left\|\psi_{\eta}(\alpha, x)-\psi_{\eta_{\varepsilon}}(\alpha, x)\right\|_{L_{p}(\mu)}$ which follows from definition of regularity of $\mu]$. Because $\left\|U_{\Lambda}\left(\varphi, \psi_{\eta_{\varepsilon}}\right)\right\|_{L_{2}\left(\mu_{0}^{\partial \Lambda} \otimes \mu\right)}$ is uniformly bounded in $\varepsilon$ [this bound is easily obtainable from definition of regularity of $\mu$ in each particular case of $\left.\psi_{\eta_{\varepsilon}}(\alpha, x)\right]$, we can conclude that $U_{\Lambda}\left(\varphi, \psi_{\eta_{\varepsilon}}\right) \underset{\varepsilon \rightarrow 0}{\longrightarrow} U_{\Lambda}\left(\varphi, \psi_{\eta}\right)$ in $L_{2}\left(\mu_{0}^{\partial \Lambda} \otimes \mu\right)$.

iii) Follows from ii) by standard arguments, e.g. [17], due to the fact that $U_{\Lambda}\left(\varphi, \psi_{\eta_{\varepsilon}}\right)(\varepsilon \geqq 0)$ are nonnegative.

Definition 1.2.1. The cylinder function $F:\left(\mathscr{S}^{\prime}, \mathscr{B}\right) \rightarrow \mathbb{R}$ is called increasing if and only if $F(\varphi)=\hat{F}\left(\varphi\left(f_{1}\right), \ldots, \varphi\left(f_{n}\right)\right)$, where $f_{i} \geqq 0, f_{i} \in \mathscr{S}, i=1, \ldots, n(n \in \mathbb{N})$, and $\hat{F}: \mathbb{R}^{n} \rightarrow \mathbb{R}$ is increasing in each variable.

Lemma 1.2.2 (correlation inequalities). i) $\mu_{\Lambda, \psi_{\eta}} \in \mathrm{FKG}$, i.e. for arbitrary increasing cylinder functions

$$
F_{1}, F_{2} \in L_{2}\left(\mu_{\Lambda, \psi_{\eta}}\right) ; \quad \mu_{\Lambda, \psi_{\eta}}\left(F_{1}, F_{2}\right) \geqq 0,
$$

and consequently for all functions $F_{i} \in L_{2}\left(\mu_{A, \psi_{\eta}}\right), i=1,2$, which can be obtained as the $L_{2}\left(\mu_{\Lambda, \psi_{\eta}}\right)$ limits of increasing cylinder functions from $L_{2}\left(\mu_{\Lambda, \psi_{\eta}}\right)$.

ii) If $\psi_{\eta}(\alpha, x)=\psi_{\eta}(-\alpha, x)$ and $d \varrho(\alpha)=d \varrho(-\alpha)$, then

$$
\mu_{\Lambda, \psi_{\eta}} \in \mathrm{GKS} \text {, }
$$

i.e. for all functions $F_{1}, F_{2} \in L_{2}\left(\mu_{\Lambda, \psi_{\eta}}\right)$ being the polynomials with nonnegative coefficients or the $L_{2}\left(\mu_{\Lambda, \psi_{\eta}}\right)$ limits of such polynomials

$$
\mu_{\Lambda, \psi_{\eta}}\left(F_{1}, F_{2}\right) \geqq 0 .
$$

Proof (see e.g. [21, 24]). This lemma is the corollary following from Lemma 1.2.1 and from the fact,that by definition (2.3) of $\mu_{A, \psi_{\eta}, \delta}$ as the locally perturbed ferromagnet $\mu_{\Lambda, \psi_{\eta}, \delta} \in \mathrm{FKG}$ in the case of i), and in the case of ii) because of the symmetry $\varphi \leftrightarrow-\varphi$, we have $\mu_{\Lambda, \psi_{\eta}, \delta} \in$ GKS.

\subsection{The FKG Order in the Set of Conditioned and Gibbs Regular Measures}

Definition 1.3. For two probability measures $\mu_{1}$ and $\mu_{2}$ on $\left(\mathscr{S}^{\prime}, \mathscr{B}\right)$, we say that $\mu_{1}$ precedes $\mu_{2}$ in the sense of $\mathrm{FKG}$ - we write $\mu_{1} \underset{\mathrm{FKG}}{\leq} \mu_{2}$ - if and only if for each increasing cylinder function $F$ on $\left(\mathscr{S}^{\prime}, \mathscr{B}\right)$

$$
\mu_{1}(F) \leqq \mu_{2}(F) .
$$

As one can see, the above relations define the partial order on the set of probability measures on $\left(\mathscr{S}^{\prime}, \mathscr{B}\right)$. The existence of FKG order in the sets of measures from local specification and of Gibbs measures for ferromagnetic specification has very interesting consequences: see [27, 30, 16, 22, 6, 35]. 
Lemma 1.3.1 (FKG order in the set of conditioned measures). Let $\Lambda \in \mathscr{F}$.

i) Let $\tilde{\psi}_{\eta} \equiv \tilde{\psi}_{\eta}(\alpha, x)$ be a random variable on $\left(\mathscr{S}^{\prime}, \mathscr{B}\right)$. Let $\theta_{\eta} \equiv \theta_{\eta}(\alpha, x)$ mean one of the following: $\alpha \phi_{\eta}, 0,-\left|\alpha \phi_{\eta}\right|$, where $\phi_{\eta}=\phi_{\eta}(x)$ is a random variable on $\left(\mathscr{S}^{\prime}, \mathscr{B}\right)$ independent of $\alpha$. We assume that $\psi_{\eta} \pm \alpha\left|\phi_{\eta}\right|$ and $\psi_{\eta}+\theta_{\eta}$ have the same properties as $\psi_{\eta}$ in (1.2). Then

$$
\mu_{\Lambda, \tilde{\psi}_{\eta}+\alpha\left|\phi_{\eta}\right|} \underset{\mathrm{FKG}}{\leftrightarrows} \mu_{\Lambda, \tilde{\psi}_{\eta}+\theta_{\eta}} \leqq \mu_{\text {FKG }} \mu_{\tilde{\psi}_{\eta}-\alpha\left|\phi_{\eta}\right|} .
$$

ii) If $\forall x \in \operatorname{int} \Lambda, \psi_{\eta}^{\partial \Lambda}(x) \leqq \psi_{\tilde{\eta}}^{\partial \Lambda}(x)$, then

$$
\mu_{\Lambda, \alpha \psi_{\eta}^{\partial \Lambda}} \underset{\text { FKG }}{\geqq} \mu_{\Lambda, \alpha \psi_{\tilde{\eta}}^{\partial \Lambda}} .
$$

Proof.i) We prove only the left-hand side FKG inequality. The proof of the righthand side is almost the same. Let $F \in L_{p}\left(\mu_{0}^{\partial \Lambda}\right), 2 \leqq p \leqq \infty$ be increasing [i.e. cylinder increasing or the $L_{2}\left(\mu_{0}^{\partial \Lambda}\right)$ limit of a sequence of cylinder increasing functions]. Let $\psi_{\eta}(s):=\psi_{\eta}+s \theta_{\eta}+(1-s) \alpha\left|\phi_{\eta}\right|, s \in[0,1]$. Then

$$
\begin{aligned}
& \mu_{\Lambda, \psi_{\eta}+\theta_{\eta}}(F)-\mu_{\Lambda, \psi_{\eta}+\alpha\left|\phi_{\eta}\right|}(F)=\int_{0}^{1} d s \frac{d}{d s} \mu_{\Lambda, \psi_{\eta}(s)}(F) \\
& =\int_{0}^{1} d s \mu_{\Lambda, \psi_{\eta}(s)}\left(F,-\lambda \int_{\Lambda} d_{2} x \int d \varrho(\alpha)\left(\theta_{\eta}(\alpha, x)-\alpha\left|\phi_{\eta}(x)\right|\right) e^{\psi_{\eta}(s)}: e^{\alpha \varphi}:_{0}(x)\right) .
\end{aligned}
$$

Furthermore,

$$
\theta_{\eta}(\alpha, x)-\alpha\left|\phi_{\eta}(x)\right|=\left\{\begin{array}{c}
\alpha\left(\phi_{\eta}-\left|\phi_{\eta}\right|\right) \\
-\alpha\left|\phi_{\eta}\right| \\
(-|\alpha|-\alpha)\left|\phi_{\eta}\right|
\end{array}\right\} \leqq 0 \text { for } \quad \text { for } \quad \alpha>0
$$

so because of the minus sign before $\lambda$, using the FKG inequality for $\mu_{\Lambda, \psi_{\eta}(s)}$ [Lemma 1.2.2i)], we see that the right-hand side of (3.2) is nonnegative. Hence because $F$ was arbitrary increasing it concludes the proof of i). The point ii) can be proven by the same arguments.

Lemma 1.3.2. Let the sequences $\left\{\mu_{\Lambda_{n}}^{1}\right\}_{\Lambda_{n} \in \mathscr{F}}$ and $\left\{\mu_{\Lambda_{n}}^{2}\right\}_{\Lambda_{n} \in \mathscr{F}}$ converge weakly to the measures $\mu^{1}$ and $\mu^{2}$, respectively.

If there exist subsequences indexed by $n^{\prime} \in \mathbb{N}$ such that

$$
\mu_{\Lambda_{n^{\prime}}}^{1} \lesseqgtr \mu_{\mathrm{FKG}}^{2} \mu_{\Lambda_{n^{\prime}}}^{2}
$$

then

$$
\mu^{1} \underset{\mathrm{FKG}}{\lesseqgtr} \mu^{2}
$$

Proof. Obvious.

Lemma 1.3.3 [18]. Let $\mu_{1}$ and $\mu_{2}$ be probability measures on $\left(\mathscr{S}^{\prime}, \mathscr{B}\right)$ such that for $f \in \mathscr{D}$

$$
\mu_{i} e^{\varphi(f)}<\infty, \quad i=1,2
$$


Let $\mu_{1} \underset{\mathrm{FKG}}{\lessgtr} \mu_{2}$ and $\mu_{1} \varphi(f)=\mu_{2} \varphi(f)$. Then

$$
\mu_{1}=\mu_{2}
$$

\subsection{The Exponential Bounds}

Let $\mu_{\Lambda, \psi_{\eta}, \pm}$ be the measures defined as in (2.2) but with an interaction given by

$$
U_{\Lambda, \pm}\left(\varphi, \psi_{\eta}\right):=\lambda \int_{\Lambda} d_{2} x \int d \varrho_{ \pm}(\alpha) e^{\psi_{\eta}(\alpha, x)}: e^{\alpha \varphi}:_{0}(x),
$$

where $d \varrho_{ \pm}$are those coming from the decomposition of $d \varrho$, see (2.1).

\section{Lemma 1.4.1.}

$$
\mu_{\Lambda, \psi_{\eta}+} \underset{\mathrm{FKG}}{\leqq} \mu_{\Lambda, \psi_{\eta}} \leqq \mu_{\mathrm{FKG}} \mu_{\psi_{\eta},-},
$$

so for $0 \leqq f \in \mathscr{D}_{\Lambda}(\Lambda \in \mathscr{F})$

$$
\mu_{\Lambda, \psi_{\eta}} e^{ \pm \varphi(f)} \leqq \mu_{\Lambda, \psi_{\eta}, \mp} e^{ \pm \varphi(f)} .
$$

Proof. Let $\mu_{\Lambda, \psi_{\eta}, s}$ for $s \in[0,1]$ be the measure defined as in (2.2), for the interaction $U_{\Lambda, s}\left(\varphi, \psi_{\eta}\right)$ defined as in $(2.1)$, but with $d \varrho_{s}(\alpha):=s d \varrho_{-}(\alpha)+d \varrho_{+}(\alpha)$, instead of $d \varrho$. Let $F \in L_{2}\left(\mu_{0}^{\partial \Lambda}\right)$ be increasing. Then

$$
\begin{aligned}
& \mu_{\Lambda, \psi_{\eta}}(F)-\mu_{\Lambda, \psi_{\eta},+}(F)=\int_{0}^{1} d s \frac{d}{d s} \mu_{\Lambda, \psi_{\eta}, s}(F) \\
& =\int_{0}^{1} d s \mu_{\Lambda, \psi_{\eta}, s}\left(F,-\lambda \int_{\Lambda} d_{2} x \int d \varrho_{-}(\alpha) e^{\psi_{\eta}(\alpha, x)}: e^{\alpha \varphi}:_{0}(x)\right) \geqq 0,
\end{aligned}
$$

from the fact that $\mu_{\Lambda, \psi_{\eta}, s} \in$ FKG [see Lemma 1.2.2i)].

In the same way we can prove the second FKG inequality in (4.2). The inequality (4.3) follows from (4.2) and the fact that both $\pm e^{ \pm \varphi(f)}$ (for $f \geqq 0$ ) are increasing.

Lemma 1.4.2 (The Exponential Bounds). i) Let $0 \leqq f \in \mathscr{D}_{\Lambda}(\Lambda \in \mathscr{F})$. Then

$$
\mu_{\Lambda, \psi_{\eta}} e^{ \pm \varphi(f)} \leqq e^{\frac{1}{2}\|f\|^{2}-1, \partial \Lambda}+\lambda A_{\psi_{\eta}}^{\partial \Lambda}(f),
$$

where

$$
A_{\psi_{\eta}}^{\partial \Lambda}(f):=\int_{\Lambda} d_{2} x \int d \varrho(\alpha)|\alpha| e^{\psi_{\eta}(\alpha, x)-\frac{\alpha^{2}}{2} K^{\partial \Lambda}(x, x)} G^{\partial \Lambda}(f, x) .
$$

In particular, for $\psi_{\eta}(\alpha, x)=-\left|\alpha \psi_{\eta}^{\partial \Lambda}(x)\right|$ or zero

$$
\mu_{\Lambda, \psi_{\eta}} e^{ \pm \varphi(f)} \leqq e^{\frac{1}{2}\|f\|^{2}-1, \partial \Lambda+\lambda \int_{\Lambda} d_{2} x d \varrho(\alpha)|\alpha| G^{\partial \Lambda}(f, x)} .
$$

ii) For any $f \in \mathscr{D}_{\Lambda}(\Lambda \in \mathscr{F})$

$$
\mu_{\Lambda, 0} e^{\varphi(f)} \leqq e^{\frac{1}{2}\|f\|^{2}{ }_{1}+b\|G \times f\|_{L_{1}}},
$$

where the constant $b$ can be chosen independent of $f$ and $\Lambda \in \mathscr{F}$. 
Proof. i) We prove the bound as in (4.5) for the measures $\mu_{\Lambda, \psi_{\eta}}$, and use Lemma 1.4.1 to obtain (4.5) for $\mu_{\Lambda, \psi_{\eta}}$. Let us change the integration variables in $\mu_{\Lambda, \psi_{\eta}, \mp} e^{ \pm \varphi(f)}$ as follows:

$$
\varphi(x)=\varphi^{\prime}(x) \pm G^{\partial \Lambda}(f, x) .
$$

Then taking into account that (see e.g. [21, p. 171])

$$
\frac{d \mu_{0}^{\partial \Lambda}\left(\cdot \pm G^{\partial \Lambda}(f, \cdot)\right)}{d \mu_{0}^{\partial \Lambda}}\left(\varphi^{\prime}\right)=e^{-\frac{1}{2}\|f\|^{2}-1, \partial \Lambda-\varphi^{\prime}( \pm f)},
$$

we have

$$
\mu_{\Lambda, \psi_{\eta}, \mp} e^{ \pm \varphi(f)}=e^{\frac{1}{2}\|f\|^{2}-1, \partial \Lambda} \frac{\mu_{0}^{\partial \Lambda} e^{-U_{\Lambda, \pm}\left(\varphi \mp G^{\partial \Lambda}(f, \cdot), \psi_{\eta}\right)}}{\mu_{0}^{\partial \Lambda} e^{-U_{\Lambda, \mp}\left(\varphi, \psi_{\eta}\right)}}
$$

Let

$$
F_{\mp}(s):=\frac{\mu_{0}^{\partial \Lambda} e^{-U_{\Lambda, \mp}^{s}}}{\mu_{0}^{\partial \Lambda} e^{-U_{\Lambda, \mp}}}, \quad s \in[0,1]
$$

where $U_{\Lambda, \mp}^{s}\left(\varphi \pm s G^{\partial \Lambda}(f, \cdot), \psi_{\eta}\right)$.

The functions $F_{\mp}(s)$ are differentiable and

$$
\begin{aligned}
\frac{d}{d s} F_{\mp}(s)= & \frac{\mu_{0}^{\partial \Lambda} e^{-U_{\Lambda, \mp}^{s}}\left(-\frac{d}{d s} U_{\Lambda, \mp}^{s}\right)}{\mu_{0}^{\partial \Lambda} e^{-U_{\Lambda, \mp}}} \\
= & \frac{1}{\mu_{0}^{\partial \Lambda} e^{-U_{\Lambda, \mp}} \mu_{0}^{\partial \Lambda}\left(e ^ { - U _ { \Lambda , \mp } } \left(-\lambda \int_{\Lambda} d_{2} x d \varrho_{\mp}(\alpha)\right.\right.} \\
& \left.\left.\cdot e^{\psi_{\eta}(\alpha, x) \mp s \alpha G^{\partial \Lambda}(f, x)}\left( \pm \alpha G^{\partial \Lambda}(f, x)\right): e^{\alpha \varphi}:_{0}(x)\right)\right) \geqq 0
\end{aligned}
$$

(because the integrand is nonnegative).

We have

$$
\frac{d}{d s} F_{\mp}(s)=\frac{\mu_{0}^{\partial \Lambda} e^{-U_{\Lambda, \mp}^{s}}\left(-\frac{d}{d s} U_{\Lambda, \mp}^{s}\right)}{\mu_{0}^{\partial \Lambda} e^{-U_{\Lambda, \mp}^{S}}} F_{\mp}(s) .
$$

The following inequality holds

$$
0 \leqq \frac{\mu_{0}^{\partial \Lambda} e^{-U_{\Lambda, \mp}^{s}}\left(-\frac{d}{d s} U_{\Lambda, \mp}^{s}\right)}{\mu_{0}^{\partial \Lambda} e^{-U_{\Lambda, \mp}^{s}}} \leqq \lambda \int_{\Lambda} d_{2} x d \varrho_{\mp}(\alpha)|\alpha| e^{\psi_{\eta}(\alpha, x)}{ }^{-\frac{\alpha^{2}}{2} K^{\partial \Lambda}(x, x)} G^{\partial \Lambda}(f, x) .
$$

Using (4.10) and (4.12)-(4.14) we can conclude (4.6).

Let us now prove (4.14): We consider the measures

$$
\mu_{\sigma, \mp}(\cdot):=\frac{\mu_{0}^{\partial \Lambda}\left(e^{-\sigma U_{\Lambda, \mp}^{s},}\right)}{\mu_{0}^{\partial \Lambda}\left(e^{-\sigma U_{\Lambda, \mp}^{s}}\right)}, \quad \sigma \in[0,1] .
$$

As it follows from Lemma 1.2.2i) $\mu_{\sigma, \mp} \in \mathrm{FKG}$. 
The functions

$$
B_{\mp}^{s}(\sigma):=\mu_{\sigma, \mp}\left(\frac{d}{d s}\left(U_{\Lambda, \mp}^{s}\right)\right)
$$

are differentiable in $\sigma$ and

$$
\frac{d}{d \sigma} B_{\mp}^{s}(\sigma)=\mu_{\sigma, \mp}\left(\frac{d}{d s}\left(-U_{\Lambda, \mp}^{s}\right),\left(-U_{\Lambda, \mp}^{s}\right)\right) \leqq 0
$$

which follows from FKG. From (4.17) we have

$$
\begin{aligned}
B_{\mp}^{s}(\sigma) & \leqq B_{\mp}^{s}(0) \\
& =\mu_{0}^{\partial \Lambda} \lambda \int_{\Lambda} d_{2} x d \varrho_{\mp}(\alpha)|\alpha| G^{\partial \Lambda}(f, x) e^{\psi_{\eta}(\alpha, x)-\frac{\alpha^{2}}{2} K^{\partial \Lambda}(x, x) \pm \alpha G^{\partial \Lambda}(f, x)}: e^{\alpha \varphi}:_{0}(x) .
\end{aligned}
$$

Hence (taking also into account that $e^{-|\alpha| G^{\partial \Lambda}(f, x)} \leqq 1$ ) the inequality (4.14) holds. This ends the proof of i).

ii) Let us change the variable of integration $\varphi \rightarrow \varphi+G^{\partial \Lambda}(f, \cdot)$. Then we have

$$
\begin{aligned}
\mu_{\Lambda, 0} e^{\varphi(f)} & =e^{\frac{1}{2}\|f\|^{2}-1, \partial \Lambda} \frac{\mu_{0}^{\partial \Lambda} e^{-U_{\Lambda}\left(\varphi+G^{\partial \Lambda}(f, \cdot)\right)}}{\mu_{0}^{\partial \Lambda} e^{-U_{\Lambda}(\varphi)}} \\
& =e^{\frac{1}{2}\|f\|^{2}-1, \partial \Lambda} \mu_{\Lambda, 0} e^{-\left(U_{\Lambda}\left(\varphi+G^{\partial \Lambda}(f, \cdot)\right)-U_{\Lambda}(\varphi)\right)} .
\end{aligned}
$$

But

$$
\begin{aligned}
& e^{-\left(U_{\Lambda}\left(\varphi+G^{\partial \Lambda}(f, \cdot)\right)-U_{\Lambda}(\varphi)\right)} \\
& \leqq \\
& \quad \exp \lambda \int_{\Lambda \cap\left\{G^{\partial \Lambda}(f, x)>0\right\}} d_{2} x \int d \varrho_{-}(\alpha)\left(1-e^{\alpha G^{\partial \Lambda}(f, x)}\right): e^{\alpha \varphi}:_{0}(x) \\
& \quad \cdot \exp \lambda \int_{\Lambda \cap\left\{G_{\partial \Lambda}(f, x)<0\right\}} d_{2} x \int d \varrho_{+}(\alpha)\left(1-e^{\alpha G^{\partial \Lambda}(f, x)}\right): e^{\alpha \varphi}:_{0}(x) .
\end{aligned}
$$

The first factor on the right-hand side of (4.20) is decreasing and the second is increasing, hence using FKG we obtain

$$
\begin{aligned}
\mu_{\Lambda, 0} e^{-\left(U_{\Lambda}\left(\varphi+G^{\partial \Lambda}(f, \cdot)-U_{\Lambda}(\varphi)\right)\right.} & \leqq \\
\leqq & \mu_{\Lambda, 0} \exp \left(\lambda \int_{\Lambda \cap\left\{G^{\partial \Lambda}(f, x)>0\right\}} d_{2} x d \varrho_{-}(\alpha)\left(1-e^{\alpha G^{\partial \Lambda}(f, x)}\right): e^{\alpha \varphi}:_{0}(x)\right) \\
& \cdot \mu_{\Lambda, 0} \exp \left(\lambda \int_{\Lambda \cap\left\{G^{\partial \Lambda}(f, x)<0\right\}} d_{2} x d \varrho_{+}(\alpha)\left(1-e^{\alpha G^{\partial \Lambda}(f, x)}\right): e^{\alpha \varphi}:_{0}(x)\right) .
\end{aligned}
$$

We now find the bound for the first factor on the right-hand side of (4.21) (for the second the analogous bound can be found by the same arguments). Let us consider the function of $s \in[0,1]$ :

$$
F_{1}(s):=\mu_{\Lambda, 0} \exp \left(\lambda \underset{\Lambda \cap\left\{G^{\partial \Lambda}(f, x)>0\right\}}{ } d_{2} x d \varrho_{-}(\alpha)\left(1-e^{s \alpha G^{\partial \Lambda}(f, x)}\right): e^{\alpha \varphi}:_{0}(x)\right),
$$

which for $s=1$ is equal to the first factor on the right-hand side of (4.21). We have

$$
\begin{aligned}
\frac{d}{d s} F_{1}(s):= & \mu_{\Lambda, 0}^{s}\left(\lambda \underset{\Lambda \cap\left\{G^{\partial \Lambda}(f, x)>0\right\}}{\int} d_{2} x d \varrho_{-}(\alpha)|\alpha| G^{\partial \Lambda}(f, x) e^{s \alpha G^{\partial \Lambda}(f, x)}: e^{\alpha \varphi}:_{0}(x)\right) \\
& \cdot F_{1}(s) \geqq 0,
\end{aligned}
$$


where

$$
\mu_{\Lambda, 0}^{s}(\cdot):=\frac{1}{Z_{\Lambda, s}} \mu_{\Lambda, 0}\left(\left(\exp \lambda \int_{\Lambda \cap\left\{G^{\partial \Lambda}(f, x)>0\right\}} d_{2} x d \varrho_{-}(\alpha)\left(1-e^{s \alpha G^{\partial \Lambda}(f, x)}\right): e^{\alpha \varphi}:_{0}(x)\right) \cdot\right)
$$

$Z_{\Lambda, s}$ a normalization factor.

Applying FKG (and taking into account that $\left.e^{s \alpha G^{\partial \Lambda}(f, x)} \leqq 1\right)$ we have

$$
\begin{aligned}
& \mu_{\Lambda, 0}^{s}\left({ }_{\Lambda \cap\left\{G^{\partial \Lambda}(f, x)>0\right\}} d_{2} x d \varrho_{-}(\alpha)|\alpha| G^{\partial \Lambda}(f, x) e^{s \alpha G^{\partial \Lambda}(f, x)}: e^{\alpha \varphi}:_{0}(x)\right) \\
& \leqq \mu_{\Lambda, 0,+}\left({ }_{\Lambda \cap\left\{G^{\partial \Lambda}(f, x)>0\right\}} d_{2} x d \varrho_{-}(\alpha)|\alpha| G^{\partial \Lambda}(f, x): e^{\alpha \varphi}:_{0}(x)\right) .
\end{aligned}
$$

Now using our exp bound i) at the end we have that the right-hand side of (4.25) is estimated by

$$
\lambda e^{\frac{\bar{\alpha}^{2} \lambda}{2 \pi m^{2}}} \int_{\Lambda \cap\left\{G^{\partial \Lambda}(f, x)>0\right\}} d_{2} x d \varrho_{-}(\alpha)|\alpha| G^{\partial \Lambda}(f, x),
$$

where $\bar{\alpha}=\max \{|\alpha|: \alpha \in \operatorname{supp} d \varrho\}$. Hence from that and (4.23) we have for a constant $b>0$,

$$
F_{1}(1) \leqq \exp \left(b \int_{\Lambda \cap\left\{G^{\partial \Lambda}(f, x)>0\right\}} d_{2} x d \varrho_{-}(\alpha)|\alpha| G^{\partial \Lambda}(f, x)\right) .
$$

Analogously, we can prove the following bound for the second factor $F_{2}(1)$ on the right-hand side of (4.21):

$$
F_{2}(1) \leqq \exp \left(b \int_{\Lambda \cap\left\{G^{\partial \Lambda}(f, x)<0\right\}} d_{2} x d \varrho_{+}(\alpha) \alpha\left|G^{\partial \Lambda}(f, x)\right|\right) .
$$

Combining (4.19) with (4.21), (4.26), and (4.27) we obtain

$$
\begin{aligned}
\mu_{\Lambda, 0} e^{\varphi(f)} & \leqq \exp \left(\frac{1}{2}\|f\|_{-1, \partial \Lambda}^{2}+b \int_{\Lambda} d_{2} x d \varrho(\alpha)|\alpha|\left|G^{\partial \Lambda}(f, x)\right|\right) \\
& \leqq \exp \left(\frac{1}{2}\|\mathrm{f}\|_{-1}^{2}+b^{\prime}\|G \times f\|_{L_{1}}\right) .
\end{aligned}
$$

The analogous bound for $\psi_{\eta}(\alpha, x) \equiv 0$ can be obtained for any $\psi_{\eta}(\alpha, x)=\alpha \psi_{\eta}^{\partial \Lambda}(x)$ with $\eta \equiv c$, where $c$ is a constant [with constant $b^{\prime}$ independent of $c$ for $d(\operatorname{supp} f, \partial \Lambda)$ sufficiently big].

\subsection{Exponential Clustering for Conditioned Measures}

Uniform in Volume and Boundary Conditions

Lemma 1.5.1. Let $f, g \in \mathscr{D}_{A}$ and $f \geqq 0$ or $\leqq 0, g \geqq 0$ or $\leqq 0$, then

$$
\begin{aligned}
0 & \leqq \mu_{\Lambda, \psi_{\eta}}\left(\operatorname{sign} f e^{\varphi(f)}, \operatorname{sign} g e^{\varphi(g)}\right) \\
& \leqq\left(\mu_{\Lambda, \psi_{\eta}} e^{\frac{1}{2} \varphi(f+g)}\right)^{2}\left|\frac{1}{2} \mu_{0}^{\partial \Lambda} 2 \sinh \frac{\varphi(f)}{\sqrt{2}} \cdot 2 \sinh \frac{\varphi(g)}{\sqrt{2}}\right| .
\end{aligned}
$$

Remark. We do not take the bound with $\mu_{\Lambda, \psi_{\eta}} e^{\varphi(f+g)}$ in the right-hand side because the case we are interested in is when we take $: e^{\alpha \varphi}:_{0}(x)$ instead of $e^{\varphi(g)}$.

Proof. We have

$$
\mu_{\Lambda, \psi_{\eta}}\left(e^{\varphi(f)}, e^{\varphi(g)}\right)=\frac{1}{2} \mu_{\Lambda, \psi_{\eta}} \otimes \tilde{\mu}_{\Lambda, \psi_{\eta}}\left(e^{\varphi(f)}-e^{\tilde{\varphi}(f)}\right)\left(e^{\varphi(g)}-e^{\tilde{\varphi}(g)}\right),
$$


where $\tilde{\mu}_{\Lambda, \psi_{\eta}} \equiv \mu_{\Lambda, \psi_{\eta}}$. Let us change the integration variables in the right-hand side of (5.1) as follows:

$$
\varphi(x)=\frac{1}{\sqrt{2}}(q(x)+p(x)), \quad \tilde{\varphi}(x)=\frac{1}{\sqrt{2}}(q(x)-p(x)) .
$$

Because

$$
\frac{d \mu_{0, \varphi}^{\partial \Lambda} \otimes \mu_{0, \tilde{\varphi}}^{\partial \Lambda}}{d \mu_{0, q}^{\partial \Lambda} \otimes \mu_{0, p}^{\partial \Lambda}}=1
$$

(where the superscripts denote the integration variables), it follows that

$$
\begin{aligned}
\mu_{\Lambda, \psi_{\eta}}\left(e^{\varphi(f)}, e^{\varphi(g)}\right)= & \frac{1}{2 Z_{\Lambda, \eta}^{2}} \mu_{0, q}^{\partial \Lambda} \otimes \mu_{0, p}^{\partial \Lambda}\left(e^{-\left(U_{\Lambda}\left(\cdot, \psi_{\eta}\right)+U_{\Lambda}\left(\cdot, \psi_{\eta}\right)\right)(q, p)}\right) \\
& \cdot e^{\frac{q(f)}{\sqrt{2}}} 2 \sinh \frac{p(f)}{\sqrt{2}} e^{\frac{q(g)}{\sqrt{2}}} 2 \sinh \frac{p(g)}{\sqrt{2}}
\end{aligned}
$$

where

$$
\begin{aligned}
& Z_{\Lambda, \eta}:=\mu_{0}^{\partial \Lambda} e^{-U_{\Lambda}\left(\varphi, \psi_{\eta}\right)} \\
\left(U_{\Lambda}\left(\cdot, \psi_{\eta}\right)+\tilde{U}_{\Lambda}\left(\cdot, \psi_{\eta}\right)\right)(q, p) \equiv & U_{\Lambda}\left(\varphi(q, p), \psi_{\eta}\right)+U_{\Lambda}\left(\tilde{\varphi}(q, p), \psi_{\eta}\right) \\
= & \lambda \int_{\Lambda} d_{2} x d \varrho(\alpha) e^{\psi_{\eta}(\alpha, x)}: e^{\frac{\alpha q}{\sqrt{2}}}:_{0}(x) 2: \cosh \frac{\alpha p}{\sqrt{2}}:_{0}(x) \\
\equiv & \lambda V_{\Lambda, \psi_{\eta}}(p \mid q) .
\end{aligned}
$$

Because

$$
\mu_{0, p}^{\partial \Lambda} e^{-\lambda V_{\Lambda, \psi_{\eta}}(p \mid q)}>0 \text { for } \mu_{0, q^{- \text {a.a. }}}^{\partial \Lambda} \quad q \in \mathscr{S}^{\prime},
$$

we can write (5.4) in the form

$$
\begin{aligned}
& \mu_{\Lambda, \psi_{\eta}}\left(e^{\varphi(f)}, e^{\varphi(g)}\right) \\
&=\frac{1}{2 Z_{\Lambda, \eta}^{2}} \mu_{0, q}^{\partial \Lambda} \\
& \cdot\left[e^{\frac{q(f+g)}{\sqrt{2}}}\left(\mu_{0, p}^{\partial \Lambda} e^{-\lambda V_{\Lambda, \psi_{\eta}}(p \mid q)}\right) v_{\Lambda, \psi_{\eta}}^{q}\left(2 \sinh \frac{p(f)}{\sqrt{2}} 2 \sinh \frac{p(g)}{\sqrt{2}}\right)\right],
\end{aligned}
$$

where

$$
v_{\Lambda, \psi_{\eta}}^{q}(\cdot):=\frac{\mu_{0, p}^{\partial \Lambda}\left(e^{-\lambda V_{\Lambda, \psi_{\eta}}(p \mid q)} \cdot\right)}{\mu_{0, p}^{\partial \Lambda}\left(e^{-\lambda V_{\Lambda, \psi_{\pi}}(p \mid q)}\right)}
$$

We will show that

$$
\begin{aligned}
0 & \leqq v_{\Lambda, \psi_{\eta}}^{q}\left((\operatorname{sgn} f) 2 \sinh \frac{p(f)}{\sqrt{2}}(\operatorname{sgn} g) 2 \sinh \frac{p(g)}{\sqrt{2}}\right) \\
& \leqq \mu_{0, p}^{\partial \Lambda}(\operatorname{sgn} f) 2 \sinh \frac{p(f)}{\sqrt{2}}(\operatorname{sgn} g) 2 \sinh \frac{p(g)}{\sqrt{2}} .
\end{aligned}
$$


Hence, from (5.9) and (5.7) by a simple change of integration variables [opposite to (5.2)] the lemma follows.

Let us prove (5.9): Let us consider the measures $v_{\Lambda, \psi_{\eta}}^{q \varepsilon}$, defined as $v_{\Lambda}^{q}{ }_{\psi_{\eta}}$ in (5.8) but with $q_{\varepsilon}(x):=q\left(h_{\varepsilon}(\cdot-x)\right)$, where $h_{\varepsilon} \geqq 0, h_{\varepsilon} \in C_{0}^{\infty}\left(\mathbb{R}^{2}\right), h_{\varepsilon} \stackrel{\varepsilon \rightarrow 0}{\longrightarrow} \delta_{x}$, instead of $q$. Because

$$
V_{\Lambda, \psi_{\eta}}\left(p \mid q_{\varepsilon}\right) \underset{\varepsilon \rightarrow 0}{\longrightarrow} V_{\Lambda, \psi_{\eta}}(p \mid q) \quad \text { in } \quad L_{2}\left(\mu_{0, q}^{\partial \Lambda} \otimes \mu_{0, p}^{\partial \Lambda}\right)
$$

and

$$
V_{\Lambda, \psi_{\eta}}\left(p \mid q_{\varepsilon}\right) \geqq 0
$$

it follows that

$$
v_{\Lambda, \psi_{\eta}}^{q_{\varepsilon}}(F) \underset{\varepsilon \rightarrow 0}{\longrightarrow} v_{\Lambda, \psi_{\eta}}^{q}(F)
$$

for any $F \in L_{s}\left(\mu_{0}^{\partial \Lambda}\right), 1 \leqq s \leqq \infty$.

Now we can define the lattice approximation for the measures $v_{\Lambda, \psi_{\eta}}^{q_{\varepsilon}}(\cdot)$ as in Sect. 1.2, and because of symmetry,

$$
V_{\Lambda, \psi_{\eta}}\left(p \mid q_{\varepsilon}\right)=V_{\Lambda, \psi_{\eta}}\left(-p \mid q_{\varepsilon}\right),
$$

we can prove GKS inequalities for lattice measures, and by the adequate limiting procedure we can prove GKS for $v_{\Lambda, \psi_{\eta}}^{q_{\varepsilon}}$. Using the GKS inequalities we get

$$
\begin{aligned}
& \frac{d}{d \lambda} v_{\Lambda, \psi_{\eta}}^{q_{\varepsilon}}\left(\operatorname{sgn} f \sinh \frac{p(f)}{\sqrt{2}} \operatorname{sgn} g \sinh \frac{p(g)}{\sqrt{2}}\right) \\
& =v_{\Lambda, \psi_{\eta}}^{q_{\varepsilon}}\left(\operatorname{sgn} f \sinh \frac{p(f)}{\sqrt{2}} \operatorname{sgn} g \sinh \frac{p(g)}{\sqrt{2}},-V_{\Lambda, \psi_{\eta}}\left(p \mid q_{\varepsilon}\right)\right) \leqq 0 .
\end{aligned}
$$

Hence

$$
\begin{aligned}
0 & \leqq v_{\Lambda, \psi_{\eta}}^{q_{\varepsilon}}\left(\operatorname{sgn} f \sinh \frac{p(f)}{\sqrt{2}} \operatorname{sgn} g \sinh \frac{p(g)}{\sqrt{2}}\right) \\
& \leqq \mu_{0}^{\partial \Lambda}\left(\operatorname{sgn} f \sinh \frac{p(f)}{\sqrt{2}} \operatorname{sgn} g \sinh \frac{p(g)}{\sqrt{2}}\right)
\end{aligned}
$$

and using (5.12) we obtain (5.9).

Remark. We can also prove this lemma by taking first the lattice approximation of (5.1) and then using the lattice version of this lemma proved in [35].

\section{Existence and Uniqueness}

In this section we prove our first main result (see Sect. 0.3): 1) Existence of exactly one point in $\mathscr{G}(\mathscr{E}) \cap \mathscr{M}_{r}$.

Proof. It is known (see $[17,2])$ that the limit $\mu \equiv \lim _{\mathscr{F}_{0}} E_{\Lambda^{c}}^{\eta \equiv 0}$ exists. We can also prove by simple compactness arguments that $\lim _{\mathscr{F}_{0}} E_{\Lambda^{c}}^{\eta=a}$ exists for any constant $a \in \mathbb{R}$. It follows from our exponential bound Lemma 1.4.2ii), that these limits belong to 
$\mathscr{G}(\mathscr{E}) \cap \mathscr{M}_{r}$. Having any measure $\mu \in \mathscr{G}(\mathscr{E}) \cap \mathscr{M}_{r}$, we know that $\lim _{\mathscr{F}_{0}} E_{\Lambda^{c}}^{\eta}$ exist for $\mu$-a.a. $\eta \in \mathscr{S}^{\prime}$ (e.g. $\left.[15,30]\right)$. In order to show that $\mathscr{G}(\mathscr{E}) \cap \mathscr{M}_{r}$ consists of the unique point, it suffices to prove that $\lim _{\mathscr{F}_{0}} E_{\Lambda^{c}}^{\eta}(F)$ are independent of $\eta$ for $\mu$-a.a. $\eta \in \mathscr{S}^{\prime}$ and any $\mu \in \mathscr{G}(\mathscr{E}) \cap \mathscr{M}_{r}$, and any continuous cylinder function $F \in L_{1}(\mu) \cap \bigcup_{\mathscr{F}_{0}} \mathscr{B}_{\Lambda}$.
Because

$$
E_{\Lambda^{c}}^{\eta} F(\varphi)=\mu_{\Lambda, \alpha \psi_{\eta}^{\partial \Lambda}} F\left(\varphi+\psi_{\eta}^{\partial \Lambda}\right)
$$

and $\psi_{\eta}^{\partial \Lambda}(x) \rightarrow 0$ locally uniformly as $d(0, \partial \Lambda) \rightarrow \infty$ [see Lemma 1.1.2i)] for $\mu$-a.a. $\eta \in \mathscr{S}^{\prime}$, so it suffices to prove that the $\operatorname{limit} \lim _{\mathscr{F}_{0}} \mu_{\Lambda, \alpha \psi_{\eta} \partial \Lambda}$, which exists for $\mu$-a.a. $\eta$, is
independent of $\eta, \mu$-a.e. It follows from

Lemma 2.1. For $\mu$-a.a., $\eta \in \mathscr{S}^{\prime}$

$$
\lim _{\mathscr{F}_{0}} \mu_{\Lambda,+\alpha\left|\psi_{\eta}^{\partial \Lambda}\right|}=\lim _{\mathscr{F}_{0}} \mu_{\Lambda,-\alpha\left|\psi_{\eta}^{\partial \Lambda}\right|} .
$$

If (2) holds then, because from Lemma 1.3.1i) we have

$$
\begin{gathered}
\mu_{\Lambda, \alpha\left|\psi_{\eta}^{\partial \Lambda}\right|} \leqq \mu_{\Lambda, \alpha \psi_{\eta}^{\hat{D}}} \leqq \mu_{\Lambda,-\alpha\left|\psi_{\eta}^{\partial \Lambda}\right|}, \\
\mu_{\Lambda, \alpha\left|\psi_{\eta}^{\partial \Lambda}\right|} \leqq \mu_{\Lambda, 0} \leqq \mu_{\Lambda,-\alpha\left|\psi_{\eta}^{\partial \Lambda}\right|} .
\end{gathered}
$$

Hence using Lemma 1.3.2, we can conclude that

$$
\lim _{\mathscr{F}_{0}} \mu_{\Lambda, \alpha \psi_{\eta}^{2} \Lambda}=\lim _{\mathscr{F}_{0}} \mu_{\Lambda, 0},
$$

so

$$
\lim _{\mathscr{F}_{0}} E_{\Lambda^{c}}^{\eta}=\lim _{\mathscr{F}_{0}} \mu_{\Lambda, 0}, \quad \mu \text {-a.e. }
$$

for any $\mu \in \mathscr{G}(\mathscr{E}) \cap \mathscr{M}_{r}$. This ends the proof of uniqueness.

Proof of Lemma 2.1. Because $\left\{\mu_{\Lambda, \alpha \psi_{\eta} \partial_{1}}\right\}_{\Lambda \in \mathscr{F}_{0}}$ converges for $\mu$-a.a. $\eta \in \mathscr{S}^{\prime}$, so from (3) if we prove that for $f \in \mathscr{D}, f \geqq 0$,

$$
\lim _{\mathscr{F}_{0}}\left(\mu_{\Lambda,-\alpha\left|\psi_{\eta}^{\partial_{\Lambda}}\right|} \varphi(f)-\mu_{\Lambda, \alpha\left|\psi_{\eta}^{\partial \Lambda}\right|} \varphi(f)\right)=0
$$

for $\mu$-a.a. $\eta$ and for a dense in $\mathscr{H}_{-1}$ countable set $\left\{f_{i} \in \mathscr{D}\right\}_{i \in \mathbb{N}}$

$$
\mu_{\Lambda, \pm \alpha\left|\psi_{\eta}^{\partial \Lambda}\right|} e^{\varphi\left(f_{i}\right)}<c\left(\eta, f_{i}\right)<\infty \quad\left(\Lambda \in \mathscr{F}_{0}\right)
$$

for $\mu$-a.a. $\eta$ [where $c\left(\eta, f_{i}\right)$ is a constant independent of $\Lambda$ ], then we'll conclude that the limits in (2) exist and are equal for $\mu$-a.a. $\eta \in \mathscr{S}^{\prime}$. (Here we use Lemma 1.3.2 and Lemma 1.3.3.)

Let us prove (7): We'll prove that for $f \in \mathscr{D}, f \geqq 0$,

$$
\lim _{\mathscr{F}_{0}}\left|\mu_{\Lambda, \pm \alpha\left|\psi_{\eta}{ }^{\Lambda}\right|} \varphi(f)-\mu_{\Lambda,-\left|\alpha \psi_{\eta}{ }^{\partial_{1}}\right|} \varphi(f)\right|=0 .
$$

We have with $\psi_{\eta}(s):=(-s \alpha-(1-s)|\alpha|)\left|\psi_{\eta}^{\partial \Lambda}(x)\right|, s \in[0,1]$,

$$
\begin{aligned}
& \mu_{\Lambda,-\alpha\left|\psi_{\eta}^{0} \Lambda\right|} \varphi(f)-\mu_{\Lambda,-\left|\alpha \psi_{\eta}^{\partial \Lambda}\right|} \varphi(f)=\int_{0}^{1} d s \frac{d}{d s} \mu_{\Lambda, \psi_{\eta}(s)} \varphi(f)
\end{aligned}
$$

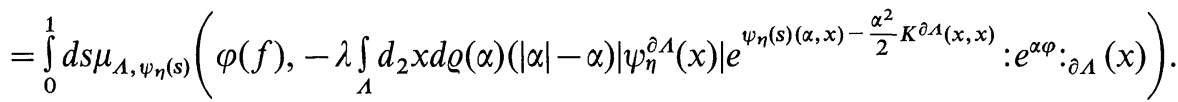


From Lemma 1.5.1 (uniform clustering), we have

$$
\begin{aligned}
& \left|\mu_{\Lambda, \psi_{\eta}(s)}\left(\varphi(f),: e^{\alpha \varphi}:_{\partial \Lambda}(x)\right)\right| \\
& \quad \leqq\left(\mu_{\Lambda, \psi_{\eta}(s)}: e^{\frac{\alpha \varphi}{2}}:_{\partial \Lambda}(x)\right)^{2} \frac{1}{\sqrt{2}}\left|\mu_{0}^{\partial \Lambda} \varphi(f) 2: \sinh \frac{\alpha \varphi}{\sqrt{2}}:_{\partial \Lambda}(x)\right| .
\end{aligned}
$$

Because of FKG order [Lemma 1.3.1i)], for $\alpha<0$,

$$
\mu_{\Lambda, \psi_{\eta}(s)}: e^{\frac{\alpha \varphi}{2}}:_{\partial \Lambda}(x) \leqq \mu_{\Lambda,-\left|\alpha^{\prime} \psi_{\eta}^{\partial \Lambda}(\cdot)\right|}: e^{\frac{\alpha \varphi}{2}}:_{\partial \Lambda}(x) \leqq \mathrm{const},
$$

where we have used Lemma 1.4.2i) [exp bound (4.7)], together with the adequate limiting procedure (because of : : $\left.\partial_{\alpha}\right)$. From (10), (11), and (12) follows

$$
\begin{aligned}
0 & \leqq \mu_{\Lambda,-\alpha\left|\psi_{\eta}^{\partial \Lambda}\right|} \varphi(f)-\mu_{\Lambda,-\left|\alpha \psi_{\eta}^{\partial \Lambda}\right|} \varphi(f) \\
& \leqq \text { const } \int_{\Lambda} d_{2} x d \varrho_{-}(\alpha) \alpha^{2} e^{\left|\alpha \psi_{\eta}^{\partial \Lambda}(x)\right|-\frac{\alpha^{2}}{2} K^{\partial \Lambda}(x, x)}\left|\psi_{\eta}^{\partial \Lambda}(x)\right| G^{\partial \Lambda}(f, x) .
\end{aligned}
$$

Let us now take the countable bases $\left\{\Omega_{n^{\prime}} \subset \Lambda_{n^{\prime}} \in \mathscr{F}\right\}_{n^{\prime} \in \mathbb{N}}$ as in Lemma 1.1.2i). Then we conclude from this lemma that

$$
\begin{aligned}
& \int_{\Omega_{n^{\prime}}} d_{2} x d \varrho_{-}(\alpha) \alpha^{2} e^{\left|\bar{\alpha} \psi_{\eta}^{\partial \Lambda}(x)\right|-\frac{\alpha 2}{2} K^{\partial \Lambda}(x, x)}\left|\psi_{\eta}^{\partial \Lambda}(x)\right| G^{\partial \Lambda}(f, x) \\
& \quad \leqq \bar{\alpha}^{2} \sup _{x \in \Omega_{n^{\prime}}}\left(\left|\psi_{\eta}^{\partial \Lambda}(x)\right| e^{\bar{\alpha}\left|\psi_{\eta}^{\partial \Lambda}(x)\right|}\right)\|G \times f\|_{L_{1}} \underset{n^{2} \rightarrow \infty}{\longrightarrow} 0
\end{aligned}
$$

for $\mu$-a.a. $\eta \in \mathscr{S}^{\prime}$.

The second component has estimation, with $1<p$,

$$
\begin{aligned}
& \int_{\Lambda_{n^{\prime}} \backslash \Omega_{n^{\prime}}} d_{2} x d \varrho_{-}(\alpha) \alpha^{2}\left|\psi_{\eta}^{\partial \Lambda}(x)\right| e^{|\alpha| \psi_{\eta}^{\partial \Lambda}(x) \mid-\frac{\alpha^{2}}{2} K^{\partial \Lambda}(x, x)} G^{\partial \Lambda}(f, x) \\
& \quad \leqq \int_{\Lambda_{n^{\prime}} \backslash \Omega_{n^{\prime}}} d_{2} x d \varrho_{-}(\alpha)|\alpha| \frac{1}{p-1} e^{p\left|\alpha \psi_{\eta}^{\partial \Lambda}(x)\right|-\frac{\alpha^{2}}{2} K^{\partial \Lambda}(x, x)} G^{\partial \Lambda}(f, x) .
\end{aligned}
$$

We have from regularity of $\mu$, if $p^{2}<2$,

$$
\begin{aligned}
& \mu \int_{\Lambda_{n^{\prime} \backslash \Omega_{n^{\prime}}}} d_{2} x d \varrho_{-}(\alpha)|\alpha| \frac{1}{p-1} e^{p\left|\alpha \psi_{\eta}^{\partial \Lambda}(x)\right|-\frac{\alpha^{2}}{2} K^{\partial \Lambda}(x, x)} G^{\partial \Lambda}(f, x) \\
& \quad \leqq \int_{\Lambda_{n^{\prime}} \backslash \Omega_{n^{\prime}}} d_{2} x d \varrho_{-}(\alpha)|\alpha| \frac{1}{p-1} e^{\frac{p^{2}-1}{2} \alpha^{2} K^{\partial \Lambda}(x, x)+b p|\alpha| \cdot \text { const }} G^{\partial \Lambda}(f, x) \\
& \quad \leqq \text { const }\|f\|_{\infty} e^{-m_{0} d\left(0, \partial \Omega_{n^{\prime}}\right)}\left|\Lambda_{n^{\prime}} \backslash \Omega_{n^{\prime}}\right|
\end{aligned}
$$

so for the sequence of $\left\{\Omega_{n^{\prime}}, \Lambda_{n^{\prime}}\right\}$ as in Lemma 1.1.2i) the right-hand side of (16) goes to zero as $n^{\prime} \rightarrow \infty$. Hence there exists the subsequence, for which the left-hand side of (15) goes to zero for $\mu$-a.a. $\eta \in \mathscr{S}^{\prime}$. Analogously, we can prove (9) for the second case. This ends the proof of (7).

In order to prove (8) it suffices to consider this bound for $f \geqq 0$ and for $f \leqq 0$ continuous with compact support. Then from Lemma 1.4.2i) we have

$$
\mu_{\Lambda, \pm \alpha\left|\psi_{\eta}^{\partial \Lambda}\right|} e^{\mp \varphi(f)} \leqq e^{\frac{1}{2}\|f\|^{2}-1, \partial \Lambda}+\lambda A_{\left|\alpha \psi_{\eta}^{\partial \Lambda}\right|}(|f|),
$$

with

$$
A_{\mid \alpha \psi_{\eta}^{\partial \Lambda}}^{\partial \Lambda}(|f|)=\int_{\Lambda} d_{2} x d \varrho(\alpha)|\alpha| e^{\left|\alpha \psi_{\eta}^{\partial \Lambda}(x)\right|-\frac{\alpha^{2}}{2} K^{\partial \Lambda}(x, x)} G^{\partial \Lambda}(|f|, x) .
$$


Because $A_{\mid \alpha \psi_{\eta} \partial(}^{\partial \Lambda}(|f|)$ converges in $L_{1}(\mu)$ to $\int d \varrho(\alpha)|\alpha|\|G \times f\|_{L 1}$, for some subsequence of $\Lambda^{\prime}$ 's, $A_{\mid \alpha \psi_{\eta} \partial \lambda}^{\partial \Lambda}(|f|)$ are bounded for $\mu$-a.a. $\eta$. Hence for any countable family of continuous functions with compact support the bound (8) holds (for some countable base $\left.\mathscr{F}_{0}\right)$.

\section{The Global Markov Property}

Let $\mu$ be the unique measure in $\mathscr{G}(\mathscr{E}) \cap \mathscr{M}_{r}$. Let $Q \subset \mathbb{R}^{2}$ be an unbounded set with piecewise $C^{1}$-boundary $\partial \Lambda$. We now show that for any $F \in \mathscr{B}_{Q} \cap L_{1}(\mu)$,

$$
E_{\mu}\left(F \mid \mathscr{B}_{Q^{c}}\right) \in \mathscr{B}_{\partial Q},
$$

i.e. that $\mu$ has GMP.

As we have stated in the introduction, the GMP problem is equivalent to the uniqueness problem for conditioned specifications

$$
\mathscr{E}_{Q, \tilde{\eta}}:=\left\{E_{(\Lambda \cap Q)}^{\eta}, \Lambda \in \mathscr{F}\right\}_{\eta|\hat{o} Q=\tilde{\eta}| \partial Q},
$$

which must be solved for $\mu$-a.a. $\tilde{\eta} \in \mathscr{S}^{\prime}$. Thus, the proof of GMP for the unique $\mu \in \mathscr{G}(\mathscr{E}) \cap \mathscr{M}_{r}$ will be carried out analogously as in Sect. 2. (uniqueness).

Remark. For our purposes it is sufficient that $\mathscr{F}$ is countable and contains a base $\mathscr{F}_{0}$ which invades $\mathbb{R}^{2}$. The measures $E_{(\Lambda \cap Q)^{c}}^{\eta}$ depend on $\eta$ through the functions $\psi_{\eta}^{\partial(\Lambda \cap Q)}(x)$. Let

$$
\begin{aligned}
& \psi_{\eta \mid \partial Q}^{\partial(Q \cap \Lambda)}(x):=\int_{\Lambda \cap \partial Q} d z P_{\partial(Q \cap \Lambda)}(x, z) \eta(z) \\
& \psi_{\eta \mid \partial \Lambda}^{\partial(Q \cap \Lambda)}(x):=\int_{Q \cap \partial \Lambda} d z P_{\partial(Q \cap \Lambda)}(x, z) \eta(z)
\end{aligned} \text { for } \quad x \in \operatorname{int}(\Lambda \cap Q)
$$

(in the sense of Sect. 1.1). We have that

$$
\psi_{\eta}^{\partial(Q \cap \Lambda)}(x)=\psi_{\eta \mid \partial Q}^{\partial(Q \cap \Lambda)}(x)+\psi_{\eta \mid \partial \Lambda}^{\partial(Q \cap \Lambda)}(x), \quad x \in \operatorname{int}(\Lambda \cap Q),
$$

where the sum means the sum of two functions in $L_{p}(\mu), 1 \leqq p<\infty$. Under the above notation (3), the symbolic expression $\eta_{\mid \partial Q}=\tilde{\eta}_{\mid \partial Q}$ in (2) means the equality

$$
\psi_{\eta_{\mid \hat{\mid} Q} Q}^{\partial(Q \cap \Lambda)}(x)=\psi_{\tilde{\eta}_{\mid \hat{\mid} Q}}^{\partial(Q \cap \Lambda)}(x)
$$

for $d_{2} x$-a.a. $x \in \operatorname{int} \Lambda \cap Q$ (in fact, for a countable dense set in $Q \cap \Lambda$ because the Bochner integral used in the definition of measure $\mu_{\Lambda, \psi_{\eta}}$ can be approximated by adequate sums).

Proof of GMP. We need to show that the limits

$$
\lim _{\mathscr{F}_{0}} E_{(\Lambda \cap Q)}^{\eta} \mid \eta_{\mid \partial Q}=\tilde{\eta}_{\mid \partial Q}
$$

(which exists for $\mu$-a.a. $\tilde{\eta}$ from the martingale convergence theorem) are independent of the sequences $\eta_{Q \cap \partial \Lambda}\left(\Lambda \in \mathscr{F}_{0}\right)$ for $\mu$-a.a. $\tilde{\eta} \in \mathscr{S}^{\prime}$.

Because from Lemma 1.1.2ii) we have that $\left|\psi_{\eta}^{\partial Q}(x)-\psi_{\eta}^{\partial(Q \cap \Lambda)}(x)\right| \underset{\Lambda \uparrow \mathbb{R}^{2}}{\longrightarrow} 0$ for $x \in \operatorname{int} Q$, it suffices to prove that the following limits (which exist $\mu$-a.e.) are independent of $\left\{\eta_{Q \cap \partial \Lambda}: \Lambda \in \mathscr{F}_{0}\right\} \mu$-a.e.,

$$
\lim _{\mathscr{F}_{0}} \mu_{\Lambda \cap Q, \alpha \psi_{\eta}^{Q}(\Lambda \cap Q)} .
$$


In order to do that it suffices (analogous to Sect. 2) to show that

$$
\lim _{\mathscr{F}_{0}} \mu_{\Lambda \cap Q, \alpha \psi_{\eta} \partial Q+\alpha\left|\delta \psi_{\eta}^{\partial \Lambda} \cap Q\right|}=\lim _{\mathscr{F}_{0}} \mu_{\Lambda \cap Q, \alpha \psi_{\eta}^{\partial Q}-\alpha \mid \delta \psi_{\eta}^{\partial \Lambda \cap Q \mid} .}
$$

Because of FKG order (Lemma 1.3.1) we have

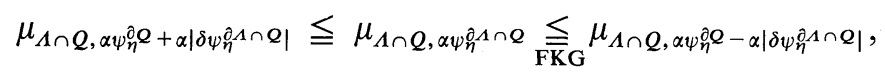

where

$$
\delta \psi_{\eta}^{\partial \Lambda \cap Q}(x):=\psi_{\eta}^{\partial \Lambda \cap Q}(x)-\psi_{\eta}^{\partial Q}(x) .
$$

So from (9) using Lemma 1.3.2 and Lemma 1.3.3 it suffices to show that for $f \in \mathscr{D}$, $f \geqq 0$

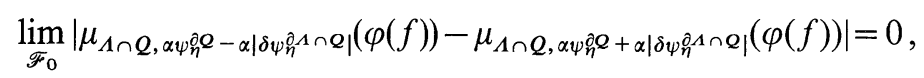

and for the total countable set $\left\{f_{i} \in \mathscr{D}_{Q}, f_{i} \geqq 0\right.$ and for $\left.f_{i} \leqq 0\right\}$,

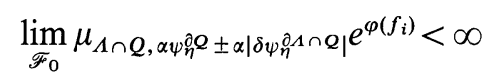

for $\mu$-a.a. $\eta \in \mathscr{S}^{\prime}$.

Let us prove (11): We have for $f \geqq 0, f \in \mathscr{D}_{Q}$ analogous to Sect. 2

$$
\begin{aligned}
& \left|\mu_{\Lambda \cap Q, \alpha \psi_{\eta}^{\partial} Q \pm \alpha\left|\delta \psi_{\eta}^{\partial \partial} \Lambda \cap Q\right|}(\varphi(f))-\mu_{\Lambda \cap Q, \alpha \psi_{\eta}^{\partial Q Q}-\left|\alpha \delta \psi_{\eta}^{\partial \Lambda} \cap Q\right|}(\varphi(f))\right| \\
& \leqq \lambda \int_{\Lambda \cap Q} d_{2} x d \varrho\left(\alpha^{\prime}\right)\left(\left|\alpha^{\prime}\right| \pm \alpha^{\prime}\right)\left|\delta \psi_{\eta}^{\partial \Lambda \cap Q}(x)\right| \\
& \cdot e^{\alpha^{\prime} \psi_{\eta}^{\partial Q} Q(x)+\left|\alpha^{\prime} \delta \psi_{\eta}^{\partial \Lambda} \cap Q(x)\right|-\frac{\alpha^{\prime 2}}{2} K^{\partial \Lambda \cap \cap Q}(x, x)} \\
& \cdot \sup _{s \in[0,1]}\left(\mu_{\Lambda \cap Q, \alpha \psi_{\eta}^{\partial Q} Q+( \pm s \alpha-(1-s)|\alpha|) \mid \delta \psi_{\eta}^{\partial \Lambda \cap Q \mid}}\left(: e^{\frac{\alpha^{\prime} \varphi}{2}}: \partial(\Lambda \cap Q)(x)\right)\right)^{2} \\
& \cdot\left|\alpha^{\prime}\right| G^{\partial \Lambda \cap Q}(f, x) \text {, }
\end{aligned}
$$

where we have used exponential clustering (Lemma 1.5.1).

From FKG order and exp bound [Lemma 1.4.2i)] we have

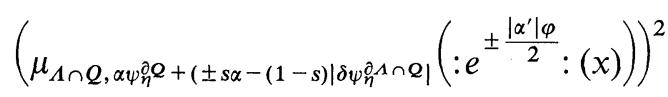

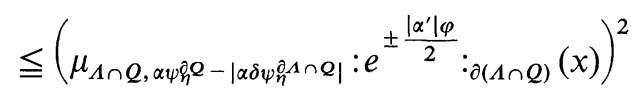

$$
\begin{aligned}
& \leqq e^{\lambda\left|\alpha^{\prime}\right| A_{\eta}^{\partial \Lambda \cap} Q_{(x)}},
\end{aligned}
$$

where

$$
\begin{aligned}
A_{\eta}^{\partial(Q \cap \Lambda)}(x): & =\int_{\Lambda \cap Q} d_{2} y d \varrho(\alpha)|\alpha| e^{\alpha \psi_{\eta}^{\partial Q}(y)-\frac{\alpha^{2}}{2} K^{\partial Q \cap \Lambda \Lambda}(y, y)} G^{\partial \Lambda \cap Q}(x, y) \\
& \leqq \int_{Q} d_{2} y d \varrho(\alpha)|\alpha| e^{\alpha \psi_{\eta}^{\partial Q}(x)-\frac{\alpha^{2}}{2} K^{\partial Q Q}(y, y)} G^{\partial Q}(x, y) \\
& =: A_{\eta}^{\partial Q}(x) .
\end{aligned}
$$


So we have from (13)-(15)

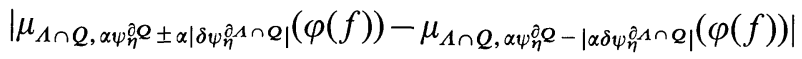

$$
\begin{aligned}
& \leqq \lambda 2 \bar{\alpha}^{2} \int_{\Lambda \cap Q} d_{2} x d \varrho(\alpha)\left|\delta \psi_{\eta}^{\partial \Lambda \cap Q}(x)\right| \\
& \cdot e^{\alpha \psi_{\eta}^{\partial Q} Q(x)+\left|\alpha \delta \psi_{\eta}^{\partial \Lambda \cap Q} Q_{(x)}\right|-\frac{\alpha^{2}}{2} K^{\partial \Lambda \cap \cap Q}(x, x)} e^{\lambda \bar{\alpha} A_{\eta}^{\partial} Q_{(x)}} G^{\partial Q \cap \Lambda}(f, x) \\
& \geqq 2 \lambda \bar{\alpha}^{2}\left(\int_{\Lambda \cap Q} d_{2} x\left|\delta \psi_{\eta}^{\partial \Lambda \cap Q}(x)\right|^{s} G^{\partial Q \cap \Lambda}(f, x)^{\frac{s}{q}}\right)^{\frac{1}{s}}
\end{aligned}
$$

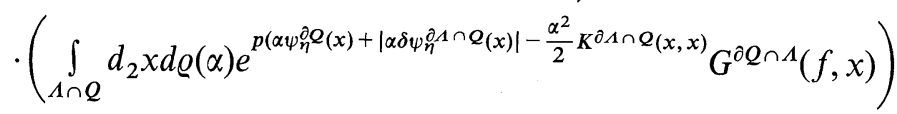

$$
\begin{aligned}
& \cdot\left(\int_{Q} d_{2} x e^{r \lambda \bar{\alpha} A_{\eta}^{\partial Q}(x)} G^{\partial Q}(f, x)^{\frac{r}{q}}\right)^{\frac{1}{r}}
\end{aligned}
$$

where $\frac{1}{p}+\frac{1}{q}=1$ with $1<p<2$ and $\frac{1}{r}+\frac{1}{s}=\frac{1}{q}$.

The first factor on the right-hand side of (16) goes to zero: For $\left\{\Omega_{n}, \Lambda_{n}\right\}_{n \in \mathbb{N}}$ as in Lemma 1.1.2ii) we have

$$
\begin{aligned}
& \int_{\Lambda_{n} \cap Q} d_{2} x\left|\delta \psi_{\eta}^{\partial Q \cap \Lambda_{n}}(x)\right|^{s} G^{\partial Q \cap \Lambda_{n}}(f, x)^{s / q} \\
& \quad=\left(\int_{\Omega_{n} \cap Q}+\int_{\left(\Lambda_{n} \backslash \Omega_{n}\right) \cap Q}\right) d_{2} x\left|\delta \psi_{\eta}^{\partial Q \cap \Lambda_{n}}(x)\right|^{s} G^{\partial Q \cap \Lambda_{n}}(f, x)^{s / q} .
\end{aligned}
$$

The first component on the right-hand side of (17) goes to zero from Lemma 1.1.2ii). The second goes to zero in $L_{1}(\mu)$ - it follows from exponential decay of $G^{\partial Q \cap \Lambda_{n}}$ uniform in $\Lambda_{n}$ and the fact that $\int_{\left(\Lambda_{n} \mid \Omega_{n}\right) \cap Q} \mu\left|\delta \psi_{\eta}^{\partial Q \cap \Lambda_{n}}(x)\right|^{s} d_{2} x$ has the bound proportional to the $\left|\partial\left(\Lambda_{n} \backslash \Omega_{n}\right) \cap Q\right|$, which grows by our assumptions at most as a power of distance $d\left(\partial \Lambda_{n}, \Omega_{n}\right)=\frac{1}{2} d\left(0, \partial \Lambda_{n}\right)$. For the second factor on the right-hand side of (16) we have (using the regularity of $\mu$ ),

$$
\begin{aligned}
& \mu \int_{\Lambda \cap Q} d_{2} x d \varrho(\alpha) e^{p\left(\alpha \psi_{\eta}^{\partial Q} Q(x)+\left|\alpha \delta \psi_{\eta}^{\partial} \Lambda \cap Q(x)\right|-\frac{\alpha^{2}}{2} K^{\partial Q Q \cap A}(x, x)\right)} G^{\partial Q \cap A}(f, x) \\
& \leqq \int_{\Lambda \cap Q} d_{2} x d \varrho(\alpha)\left[e^{\frac{p^{2}-p}{2} \alpha^{2} K^{\partial \Lambda \cap Q} Q_{(x, x)}}\right. \\
& \left.+e^{\frac{p^{2} \alpha^{2}}{2}\left(4 K^{\partial} Q(x, x)-4 K^{\partial \Lambda \cap Q} Q(x, x)+K^{\partial \Lambda \cap Q}(x, x)\right)} e^{-\frac{p \alpha^{2}}{2} K^{\partial Q} Q \cap \Lambda(x, x)}\right] \\
& \text {. const } \times G^{\partial Q \cap \Lambda}(f, x) \\
& \leqq \int_{\Lambda \cap Q} d_{2} x d \varrho(\alpha) 2 \times \text { const } \times e^{\frac{p(p-1)}{2} \alpha^{2} K^{\partial \Lambda \cap Q}(x, x)} \cdot G^{\partial Q \cap \Lambda}(f, x) \text {, }
\end{aligned}
$$

where we have used the fact, that

$$
K^{\partial Q}(x, x)=\lim _{y \rightarrow x}\left(G-G^{\partial Q}\right)(x, y) \leqq \lim _{y \rightarrow x}\left(G-G^{\partial Q \cap \Lambda}\right)(x, y)=K^{\partial Q \cap A}(x, x) .
$$


But the right-hand side of (18) is uniformly bounded in $\Lambda$, so we can choose the subsequence $\left\{\Lambda_{n} \in \mathscr{F}_{0}\right\}_{n \in \mathbb{N}}$, for which the second factor in the right-hand side of (16) weakly converges and so $\mu$-a.e. is bounded (uniformly in $\Lambda_{n}$ ).

The third factor on the right-hand side of (16) is bounded for $\mu$-a.a. $\eta \in \mathscr{S}^{\prime}$, which easily follows from the estimation

$$
A_{\eta}^{\partial Q}(x) \leqq R(\eta)\left(1+d(0, x)^{\beta}\right)
$$

with $R(\eta)$ a constant dependent on $\eta$ and $0<\beta<1$.

This ends the proof of (11).

We now prove the estimation (20) in the following two lemmas:

Lemma 3.1. Let $\left\{\Delta_{i}\right\}_{i \in \mathbb{Z}^{2}}$ be the cover of $Q$ by unit disjoint cubes with centres at the points $i \in \mathbb{Z}^{2}$. Let for $k \in \mathbb{N}$,

$$
\Omega_{k}:=\left\{\eta \in \mathscr{S}^{\prime}: \forall i \in \mathbb{Z}^{2}, \int_{\Delta_{i} \cap Q} d_{2} y\left(: e^{\bar{\alpha} \psi_{\eta}^{Q} Q(y)}:\right)^{p}<k d(0, i)^{p^{\prime}}\right\}
$$

with $1<p^{\prime}<p$ and $p(2 p-1) \bar{\alpha}^{2}<4 \pi$, where

$$
: e^{\bar{\alpha} \psi_{\eta}^{\partial} Q(y)}: \equiv e^{\bar{\alpha} \psi_{\eta}^{\partial Q} Q(y)-\frac{\bar{\alpha}^{2}}{2} K^{\partial} Q(y, y)}
$$

then

$$
\mu\left\{\bigcup_{k \in \mathbb{N}} \Omega_{k}\right\}=1
$$

Proof of Lemma 3.1. We have (using Tchebyshev's inequality and regularity of $\mu$ )

$$
\begin{aligned}
\mu\left\{\mathscr{S} \backslash \Omega_{k}\right\} & =\mu\left\{\eta \in \mathscr{S}^{\prime}: \exists i \in \mathbb{Z}^{2}, \int_{\Delta_{i} \cap Q} d_{2} y\left(: e^{\bar{\alpha} \psi_{\eta}^{\partial} Q(y)}:\right)^{p}>k d(0, i)^{p^{\prime}}\right\} \\
& \leqq \frac{1}{k^{2}} \sum_{i \in \mathbb{Z}^{2}} \frac{1}{d(0, i)^{2 p^{\prime}}} \mu \int_{\Delta_{i} \cap Q} d_{2} y\left(: e^{\bar{\alpha} \psi_{\eta}^{\partial} Q(y)}:\right)^{2 p} \\
& \leqq \operatorname{const} \frac{1}{k^{2}} \sum_{i \in \mathbb{Z}^{2}} \frac{1}{d(0, i)^{2 p^{\prime}}}\left(\int_{\Delta_{i} \cap Q} d_{2} y e^{\left(4 p^{2}-2 p\right) \cdot \frac{\bar{\alpha}^{2}}{2} K^{\partial Q} Q(y, y)}\right) \\
& <\frac{\text { const }^{\prime}}{k^{2}},
\end{aligned}
$$

because $K^{\partial Q}(y, y)$ has the singularity $-\frac{1}{2 \pi} \ln d(\partial Q, y)$, so if $p(2 p-1) \bar{\alpha}^{2}<4 \pi$, then the integrals $\int_{\Delta_{i} \cap Q} d_{2} y e^{p(2 p-1) \bar{\alpha}^{2} K^{\partial Q(y, y)}}$ exists and are uniformly bounded in $i \in \mathbb{Z}^{2}$, and because $p^{\prime}>1$ so

$$
\sum_{i \in \mathbb{Z}^{2}} \frac{1}{d(0, i)^{2 p^{\prime}}}<\infty .
$$

Hence, because the right-hand side of (22) goes to zero as $k \rightarrow \infty$, the lemma follows. 
Lemma 3.2. For $\mu$-a.a. $\eta \in \mathscr{S}^{\prime}$

$$
A_{\eta}^{\partial Q}(x)<R(\eta)\left(1+d(0, x)^{\beta}\right)
$$

with $0<\beta<1$ and $R(\eta)$ a constant dependent on $\eta \in \mathscr{S}^{\prime}$.

Proof of Lemma 3.2. We have from definition (15) of $A_{\eta}^{\partial Q}(x)$ and Hölder's inequality with $1<p<2$ and $\frac{1}{p}+\frac{1}{q}=1$,

$$
A_{\eta}^{\partial Q}(x) \leqq \sum_{i \in \mathbb{Z}^{2}} \int d \varrho(\alpha)|\alpha|\left(\int_{\Delta_{i} \cap Q} d_{2} y\left(: e^{\alpha \psi_{\eta}^{\partial Q} Q_{(y)}}:\right)^{p}\right)^{\frac{1}{p}}\left(\int_{\Delta_{i} \cap Q} d_{2} y G^{\partial Q}(x, y)^{q}\right)^{\frac{1}{q}} .
$$

Now using Lemma 3.1 with $\bar{\alpha}=\max \{\alpha: \alpha \in \operatorname{supp} d \varrho\}$ we obtain with the constants $c(\eta)$ and $a$,

$$
\begin{aligned}
A_{\eta}^{\partial Q}(x) & \leqq \sum_{i \in \mathbb{Z}^{2}} \int d \varrho(\alpha)|\alpha|\left(c(\eta) d(0, i)^{p^{\prime}}\right)^{\frac{1}{p}} a \cdot e^{-m_{0} d(x, i)} \\
& \leqq \text { const } \times\left(d(0, x)^{\frac{p^{\prime}}{p}} \sum_{i \in \mathbb{Z}^{2}} e^{-m_{0}(d(x, i))}+\sum_{i \in \mathbb{Z}^{2}} d(x, i)^{\frac{p^{\prime}}{p}} e^{-m_{0} d(x, i)}\right) \\
& \leqq R(\eta)\left(d(0, x)^{\beta}+1\right)
\end{aligned}
$$

with $R(\eta)$ a constant and $\beta=\frac{p^{\prime}}{p}<1$.

In order to end the proof of GMP we need to prove the exp bound (12): It suffices to consider the case $f \in \mathscr{D}_{Q}, f \geqq 0$ and also $f \leqq 0$, so we can use Lemma 1.4.2i), which gives

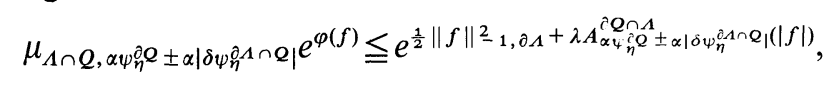

where

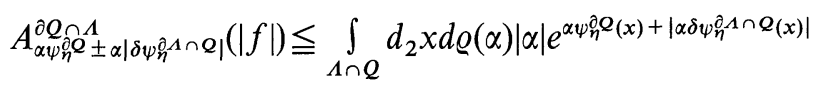

$$
\begin{aligned}
& \cdot e^{-\frac{\alpha^{2}}{2} K^{\partial \Lambda \cap \cap Q}(x, x)} G^{\partial \Lambda \cap Q}(|f|, x) .
\end{aligned}
$$

But the right-hand side of (29) converges for $\mu$-a.a. $\eta \in \mathscr{S}^{\prime}$ (eventually, if we take a subsequence) so (12) follows. This ends the proof of GMP.

Acknowledgements. The author would like to thank Prof. W. Karwowski and Dr. R. Gielerak for critical reading of the manuscript. The author is grateful to Prof. L. Streit for his kind invitation to $\mathrm{ZiF}$ of Bielefeld University and wants to express his thanks to Prof. S. Albeverio and Dr. M. Röckner for their critical remarks, which induced the improvements of a preliminary version of this work.

\section{References}

1. Accardi, L.: Local perturbation of conditional expectations. J. Math. Anal. Appl. 72, 34-63 (1979)

2. Albeverio, S., Høegh-Krohn, R.: The Wightman axioms and the mass gap for the strong interactions of exponential type in two-dimensional space-time. J. Funct. Anal. 16, 39-82 (1974) 
3. Albeverio, S., Høegh-Krohn, R.: Uniqueness and the global Markov property for Euclidean fields. The case of trigonometric interactions. Commun. Math. Phys. 68, 95-128 (1979)

4. Albeverio, S., Høegh-Krohn, R.: a) Topics in infinite dimensional analysis. In: Lecture Notes in Physics, Vol. 80. Berlin, Heidelberg, New York: Springer 1978

b) Diffusions, quantum fields, and groups of mappings, pp. 133-145. In: Lecture Notes in Mathematics, Vol. 923. Berlin, Heidelberg, New York: Springer 1982

5. Albeverio, S., Høegh-Krohn, R., Olsen, G.: The global Markov property for lattice systems. J. Multivariate Anal. 11, 599-607 (1981)

6. Bellissard, J., Høegh-Krohn, R.: Compactness and the maximal Gibbs state for random Gibbs fields on lattice. Commun. Math. Phys. 84, 297-327 (1982)

7. Bellisard, J. Picco, P.: Lattice quantum fields: Uniqueness and Markov property. Marseille Preprint 1978

8. Ciesielski, Z.: Lectures on Brownian motion, heat conduction and potential theory. Aarhus Lec. Notes No. 3, Aarhus 1965

9. Dang-Ngoc, N., Royer, G.: Markov property for extremal local fields. Proc. Am. Math. Soc. 70, 185-188 (1978)

10. Dobrushin, R.L.: The description of a random field by means of conditional probabilities and conditions of its regularity. Theor. Prob. Appl. 13, 197-224 (1968)

11. Dobrushin, R.L.: Prescribing a system of random variables by conditional distribution. Theor. Prob. Appl. 15, 458-486 (1970)

12. Dobrushin, R.L., Minlos, R.A.: Construction of the one dimensional quantum field with the aid of continuous Markov field. Funct. Anal. Its Appl. 7, No. 4, 81-82 (1973) (in Russian)

13. Dobrushin, R.L., Minlos, R.A.: Investigation of the properties of generalized Gaussian random fields, pp. 117-165. In: Problems of Mechanics and Mathematical Physics. Moscow 1976 (in Russian) [Sel. Math. Sov. 1, 215-263 (1981)]

14. Dobrushin, R.L., Shlosman, S.B.: Phases corresponding to minima of local energy. Sel. Math. Sov. 1, 317-338 (1981)

15. Fölmer, H.: Phase transition and Martin boundary. Séminaire de Probabilités Strasbourg IX. Lecture Notes in Mathematics, Vol. 536. Berlin, Heidelberg, New York: Springer 1976

16. Fölmer, H.: On the global Markov property, pp. 293-302. In: Quantum fields - algebras, processes. Streit, L. (ed.). Berlin, Heidelberg, New York: Springer 1980

17. Fröhlich, J., Park, Y.M.: Remark on exponential interactions and the quantum sine-Gordon equation in two space-time dimensions. Helv. Phys. Acta 50, 315-329 (1977)

18. Fröhlich, J., Simon, B.: Pure states for general $P(\varphi)_{2}$ theories: construction, regularity, and variational equality. Ann. Math. 105, 493-526 (1977)

19. Gielerak, R.: Verification of the global Markov property in some class of strongly coupled exponential interactions. J. Math. Phys. 24, 347-355 (1983)

20. Gielerak, R., Zegarliński, B.: Uniqueness and global almost Markov property for regularized Yukawa cases. Fortsch. Phys. 32, 1-24 (1984)

21. Glimm, J., Jaffe, A.: Quantum physics: a functional integral point of view. New York: Springer 1981

22. Goldstein, S.: Remarks on the global Markov property. Commun. Math. Phys. 74, 223-234 (1980)

23. Griffiths, R., Simon, B.: The $\left(\phi^{4}\right)_{2}$ field theory as a classical Ising model. Commun. Math. Phys. 33, 145-164 (1973)

24. Guerra, F., Rosen, L., Simon, B.: The $P(\varphi)_{2}$ Euclidean quantum field theory as classical statistical mechanics. Ann. Math. 101, 111-259 (1975)

25. Imbrie, J.Z.: Phase diagrams and cluster expansions for low temperature $P(\varphi)_{2}$ models. I. Commun. Math. Phys. 82, 261-304 (1981); II. Commun. Math. Phys. 82, 305-343 (1981)

26. Lanford, O.E. III, Ruelle, D.: Observables at infinity and states with short range correlations in statistical mechanics. Commun. Math. Phys. 13, 194-215 (1969)

27. Lebowitz, J.L., Martin-Löf, A.: On the uniqueness of the equilibrium state for Ising spin systems. Commun. Math. Phys. 25, 276-282 (1972) 
28. Nelson, E.: Construction of quantum fields from Markov fields. J. Funct. Anal. 12, 97-112 (1973)

29. Osterwalder, K., Schrader, R.: Axioms for Euclidean-Greens functions. Commun. Math. Phys. 31, 83-112 (1973); 42, 281-305 (1975)

30. Preston, Ch.: Random fields. In: Lecture Notes in Mathematics, Vol. 465. Berlin, Heidelberg, New York: Springer 1976

31. Royer, G.: Etude des champs Euclidiens sur un réseau $\mathbb{Z}^{v}$. J. Math. Pures Appl. 56, 455-478 (1977)

32. Ruelle, D.: Statistical mechanics of a one dimensional lattice gas. Commun. Math. Phys.9, 267 (1968)

33. Sukhov, Yu.M.: One parameter operator semigroup, induced by Gibbs distribution. Usp. Mat. Nauk XXV, 199-200 (1970); Application of the transfer-matrix method to the continuous systems of classical statistical mechanics. Usp. Mat. Nauk XXV, 277-278 (1970)

34. Yosida, K.: Functional analysis. Berlin, Heidelberg, New York: Springer 1965

35. Zegarliński, B.: Extremality and the global Markov property for Euclidean fields on lattice. Wrocław Preprint 1982

Communicated by K. Osterwalder

Received July 8, 1983; in revised form June 4, 1984

Note added in proof. Concerning the remarks on pp. 199 and 200: see the BiBoS paper "The Gibbs measures and partial differential equations" by the same author. 
